\title{
The number of Euler tours of random directed graphs
}

\author{
Páidí Creed* \\ School of Mathematical Sciences \\ Queen Mary, University of London \\ United Kingdom \\ P.Creed@qmul.ac.uk
}

\author{
Mary Cryan ${ }^{\dagger \ddagger}$ \\ School of Informatics \\ University of Edinburgh \\ United Kingdom \\ mcryan@inf.ed.ac.uk
}

Submitted: May 21, 2012; Accepted: Jul 26, 2013; Published: Aug 9, 2013

Mathematics Subject Classifications: 05A16, 05C30, 05C80, 68Q25

\begin{abstract}
In this paper we obtain the expectation and variance of the number of Euler tours of a random Eulerian directed graph with fixed out-degree sequence. We use this to obtain the asymptotic distribution of the number of Euler tours of a random $d$-in/d-out graph and prove a concentration result. We are then able to show that a very simple approach for uniform sampling or approximately counting Euler tours yields algorithms running in expected polynomial time for almost every $d$-in/d-out graph. We make use of the BEST theorem of de Bruijn, van Aardenne-Ehrenfest, Smith and Tutte, which shows that the number of Euler tours of an Eulerian directed graph with out-degree sequence $\mathbf{d}$ is the product of the number of arborescences and the term $\frac{1}{|V|}\left[\prod_{v \in V}\left(d_{v}-1\right) !\right]$. Therefore most of our effort is towards estimating the moments of the number of arborescences of a random graph with fixed out-degree sequence.
\end{abstract}

\section{Introduction}

\section{$1.1 \quad$ Background}

Let $G=(V, A)$ be a directed graph. An Euler tour of $G$ is any ordering $e_{\pi(1)}, \ldots, e_{\pi(|A|)}$ of the set of arcs $E$ such that for every $1 \leqslant i<|A|$, the target vertex of arc $e_{\pi(i)}$ is the source vertex of $e_{\pi(i+1)}$, and such that the target vertex of $e_{\pi(|A|)}$ is the source of $e_{\pi(1)}$. We use $\operatorname{ET}(G)$ to denote the set of Euler tours of $G$, where two Euler tours are considered

*Supported by EPSRC grants EP/F01161X/1, EP/D043905/1, and EP/I011528/1

†Supported by EPSRC grant EP/D043905/1

${ }^{\ddagger}$ Corresponding author 
to be equivalent if one is a cyclic permutation of the other. It is a well-known fact that a directed graph $G$ has an Euler tour if and only if $G$ is strongly connected and if for each $v \in V$, the in-degree and out-degree of $v$ are equal. In this paper, we are interested in the number of Euler tours of a random Eulerian directed graph with fixed out-degree sequence. Let $\mathbf{d}=\left(d_{1}, d_{2}, \ldots\right)$ be a sequence of positive integers. We let $\mathcal{G}_{\mathrm{n}}^{\mathbf{d}}$ be the space of all Eulerian directed graphs on vertex set $[n]=\{1,2, \ldots, n\}$ with out-degree sequence $\left(d_{1}, d_{2}, \ldots, d_{n}\right)$. We use $m=\sum_{v \in[n]} d_{v}$ to denote the number of arcs in a graph $G \in \mathcal{G}_{\mathrm{n}}^{\mathbf{d}}$. In the case where $d_{i}=d_{j}$ for all $i, j \in[n]$, we refer to the graphs as $d$-in/d-out graphs and denote this set by $\mathcal{G}_{\mathrm{n}}^{\mathrm{d}, \mathrm{d}}$. In this paper, we obtain asymptotic estimates for the first and second moments of the number of Euler tours of a uniformly random $G \in \mathcal{G}_{\mathrm{n}}^{\mathbf{d}}$, for any fixed out-degree vector $\mathbf{d}$ as $m-n \rightarrow \infty$.

Using the estimates of the moments, we determine the asymptotic distribution of the number of Euler tours of a random $G \in \mathcal{G}_{\mathrm{n}}^{\mathrm{d}, \mathrm{d}}$. Similar results have previously been obtained for various structures in the case of undirected regular graphs. For example, the asymptotic distribution has already been characterised for Hamiltonian cycles [12, 13, 5], 1 -factors [9], and 2-factors [11], in the case of uniformly random $d$-regular undirected graphs. In each of these results, one of the goals was to prove that the structure of interest occurs in $G$ with high probability when $G$ is chosen uniformly at random from the set of all undirected $d$-regular graphs. Since every connected $d$-in/d-out graph has an Euler tour, the existence question is not of interest for these structures. However, in the case of Hamiltonian cycles the asymptotic distribution was further used by Frieze et al. [5] to prove that very simple algorithms for random sampling and approximate counting of Hamiltonian cycles run in expected polynomial time for almost every $d$-regular graph. This paper contains analogous counting and sampling results for Euler tours of $d$-in $/ d$ out graphs for $d \geqslant 2$. We then exploit these results to show that very simple algorithms for sampling and/or counting Euler tours perform well when the input graph is drawn from $\mathcal{G}_{\mathrm{n}}^{\mathrm{d}, \mathrm{d}}$.

Our result uses a well-known relationship between the Euler tours and arborescences of an Eulerian graph. An arborescence of a directed graph $G=(V, A)$ is a rooted spanning tree of $G$ in which all arcs are directed towards the root. A generalization of the concept of an arborescence is that of a (in-directed) forest, a collection of disjoint rooted trees in $G$ where every arc in the forest is directed towards the root of its own tree, such that the collection of trees spans $V$. In this paper a forest will always be assumed to be in-directed. We will define the notation $A R B S(G)$ to denote the set of arborescences of $G$ and, for any $v \in V$, use $A R B S(G, v)$ to denote the set of arborescences rooted at $v$.

For any Eulerian directed graph $G$, the BEST Theorem (due to de Bruijn and van Aardenne-Ehrenfest [17], extending a result of Smith and Tutte [14]) reduces the problem of computing $|E T(G)|$ to the problem of computing the value $|A R B S(G, v)|$, for any vertex $v \in V$.

Theorem 1 ([14, 17]). Let $G=(V, A)$ be an Eulerian directed graph (or multi-graph) 
with out-degree sequence $\mathbf{d}$. For any $v \in V$, we have

$$
|E T(G)|=\left[\prod_{u \in V}\left(d_{u}-1\right) !\right]|A R B S(G, v)| .
$$

We remark that the proof of Theorem 1, though usually stated for simple Eulerian directed graphs, also holds for Eulerian directed multi-graphs with loops and parallel $\operatorname{arcs}^{1}$.

The above theorem enables exact counting or sampling of Euler tours of any Eulerian directed graph in polynomial time. For any given digraph $G=(V, A)$, the well-known Matrix-tree theorem shows that for any $v \in V$ the number of arborescences into $v \in V$ exactly equals the value of the $(v, v)$-cofactor of the Laplacian matrix of $G$ (see, for example, [16]). Colbourn et al. [4] gave an algorithm allowing sampling of a random arborescence rooted at $v$ to be carried out in the same time as counting all such arborescences. Hence, applying the BEST theorem stated above, the twin tasks of exact counting and uniform sampling of Euler tours of a given Eulerian digraph on $n$ vertices can be performed in the time to evaluate the determinant of an $n \times n$ matrix, which at the time of writing is $O\left(n^{c}\right)$ for $c<2.3727$ [18]. An alternative approach to sampling is presented in [10].

\subsection{Näive algorithms}

In this paper, we take a different approach and consider a very näive algorithm for sampling Euler tours of an Eulerian digraph. To describe this algorithm, it helps to introduce the concept of a transition system of an Eulerian digraph $G=(V, A)$ : for every $v \in V$, consider the set $\operatorname{In}(v)$ of arcs directed into $v$, and the set $\operatorname{Out}(v)$ of arcs directed away from $v$ (in a multi-graph we allow the possibility that $\operatorname{In}(v) \cap O u t(v) \neq \emptyset$ ). We define a pairing $P(v)$ at $v$ to be a matching of $\operatorname{In}(v)$ with $O u t(v)$. Finally we define a transition system of $G$ to be the union of a collection of pairings, one for each vertex of the graph. We let $T S(G)$ denote the set of all transition systems of $G$. If $G$ has the out-degree sequence $d_{v}: v \in V$, then $|T S(G)|=\prod_{v \in V} d_{v}$ !. Note that every Euler tour of $G$ induces a unique transition system on $G$.

Our näive sampling algorithm, presented in Figure 1 overleaf, generates a random transition system for $G$ and tests whether it induces an Euler tour.

We make two simple observations. First, observe that $\operatorname{SAmplE}\langle G=(V, A)\rangle$ generates all transition systems of $G$ with equal probability. Hence all transition systems corresponding to an Euler tour will be generated with a uniform probability (which is $\left[\prod_{v \in V} d_{v} !\right]^{-1}$ ).

\footnotetext{
${ }^{1}$ To see the extension for graphs with parallel arcs, consider the process of eliminating parallel arcs by subdividing each such arc using a new vertex. This process gives a graph with no parallel arcs, which has the same number of ETs and the same number of in-directed arborescences into any vertex $v$ from the original graph. Moreover, the new vertices, having in-degree and out-degree 1, do not alter the value of $\prod_{u \in V}\left(d_{u}-1\right)$ !. Hence we only need to extend the Theorem for directed Eulerian graphs with loops. Observe that no loop can ever belong to an arborescence, so the addition of loops does not alter the value of $|A R B S(G, v)|$. Adding loops does increase the number of ETs (if we add a loop at vertex $u$ then we can insert it into any of the $d_{u}$ "visits to $u$ " of an existing Euler tour), however, this increase is mirrored exactly by the increased value of $\prod_{u \in V}\left(d_{u}-1\right)$.
} 


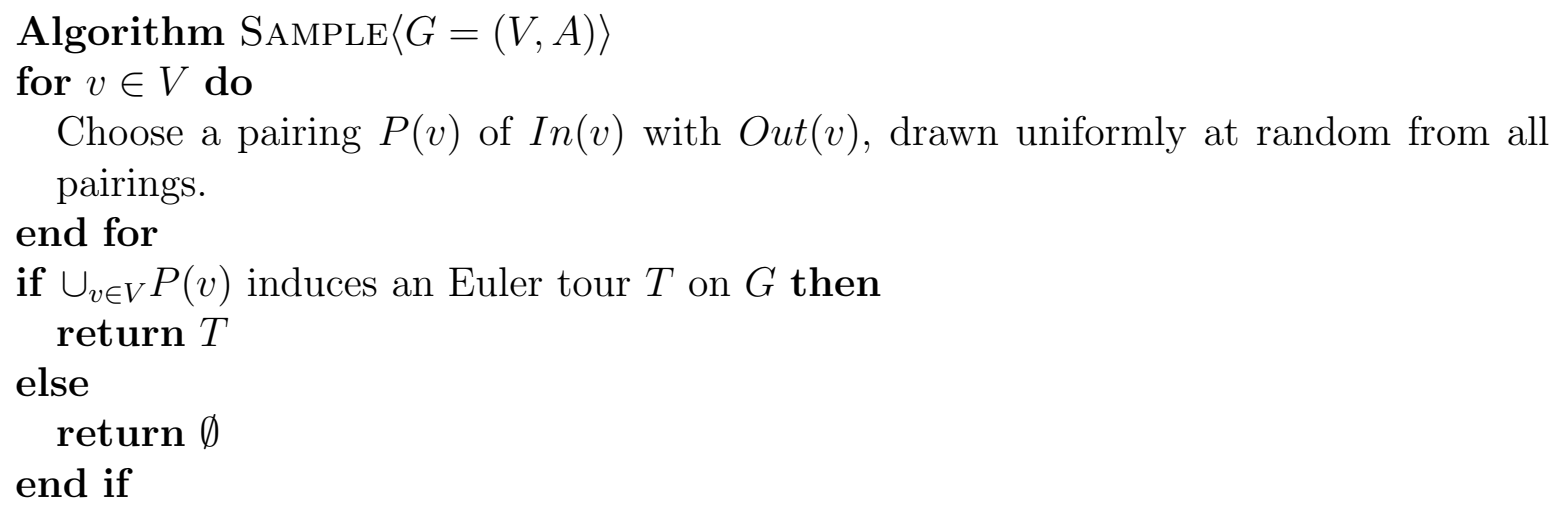

Figure 1: Algorithm SAMPLE

Second, the probability that one execution of $\operatorname{SAmpLE}\langle G=(V, A)\rangle$ returns an Euler tour is exactly $|E T(G)| /|T S(G)|=|E T(G)| \times\left[\prod_{v \in V} d_{v} !\right]^{-1}$.

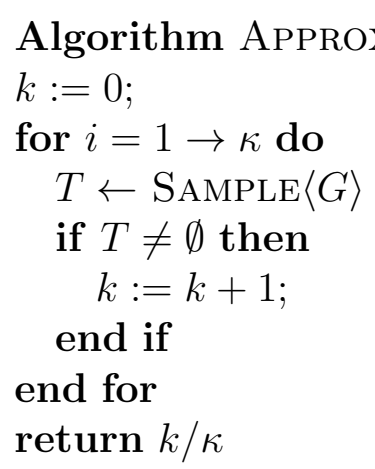

Figure 2: Algorithm Approximate

In Figure 2 overleaf, we present our simple approximate counting algorithm. We observe that for any given $\kappa \in \mathbb{N}$, that the expectation $\mathbb{E}[k / \kappa]$ of the value that is returned by Approximate $\langle G=(V, A), \kappa\rangle$ is $|E T(G)| /|T S(G)|$. However, the probability that the value returned by Approximate $\langle G=(V, A), \kappa\rangle$ will be close to $|E T(G)| /|T S(G)|$ depends both on $\kappa$ and on the value of $|E T(G)|$. If we are given a graph $G$ whereby $|E T(G)|$ is guaranteed to be larger than $p(|V|)^{-1} \prod_{v \in V} d_{v}$ !, where $p(\cdot)$ is some fixed polynomial, then by setting $\kappa$ appropriately we can guarantee that with high probability ApPROX$\operatorname{IMATE}\langle G=(V, A), \kappa\rangle$ will return a close approximation of $|E T(G)| /|T S(G)|$. However, there exist Eulerian digraphs where the number of Euler tours is only an exponentially small multiple of $\prod_{v \in V} d_{v} !$.

In this paper we consider the performance of SAMPLE and of ApPROximate on random regular Eulerian digraphs of bounded degree $d$. Our goal will be to show that as the number of vertices grows, that for some $\kappa$ polynomial in $|V|$, the probability that APPROXIMATE returns a close approximation of $|E T(G)| /|T S(G)|$ tends to 1 . This requires that we can demonstrate two things:

(a) that the expected number of Euler tours of a random Eulerian digraph of fixed 
degree $d$ on $n$ vertices is polynomially-related to $|T S(G)|=(d !)^{n}$; that is, there is some $h>0$ such that the expected number of Euler tours is greater than $n^{-h}(d !)^{n}$.

We will show this in Theorem 5 (using Theorem 3) and Corollary 6.

(b) that $|E T(G)|$ on random $d$-regular Eulerian digraphs is concentrated within a window of this expected value.

The proof of this appears in Sections 3 and 4.

Note that these natural algorithms for sampling and approximate counting of random Eulerian digraphs have previously been analysed for the case of Eulerian tournaments in [8]. This was done as part of their analysis of Euler tours on the undirected complete graph with an odd number of vertices. It does not overlap our research - tournaments are regular of degree $(n-1) / 2$.

\subsection{Our proof}

The results in this paper are of an asymptotic nature. If $a_{n}$ and $b_{n}$ are sequences of numbers, we take $a_{n} \sim b_{n}$ to mean $\lim _{n \rightarrow \infty} a_{n} / b_{n}=1$. Given a sequence of random variables $X_{n}$ and random variable $Z$, we say $X_{n}$ converges in distribution to $Z$, or $Z$ has the asymptotic distribution of $X_{n}$, if

$$
\lim _{n \rightarrow \infty} \mathbb{P}\left[X_{n} \leqslant x\right]=\mathbb{P}[Z \leqslant x]
$$

We write $X_{n} \stackrel{d}{\rightarrow} Z$ as notation for convergence in distribution.

We generate graphs in $\mathcal{G}_{\mathrm{n}}^{\mathbf{d}}$ using a directed version of the configuration model $[2,3]$. We define the configuration space $\Phi_{n}^{\mathbf{d}}$ as follows. For each $v \in[n]$, let $S_{v}$ and $T_{v}$ be disjoint $d_{v}$-sets and let $S=\cup_{v \in[n]} S_{v}$ and $T=\cup_{v \in[n]} T_{v}$. We say $S_{v}$ is the set of configuration points available for arcs leaving $v$ and $T_{v}$ is the set of points available for arcs entering $v$. A configuration $F$ is a perfect matching from $S$ to $T$ and $\Phi_{n}^{\mathbf{d}}$ is the set of all configurations. Note that $\left|\Phi_{n}^{\mathbf{d}}\right|=m$ !. Each configuration $F \in \Phi_{n}^{\mathbf{d}}$ projects to a directed multi-graph $\sigma(F)$ by identifying the elements of $S_{v}$ and $T_{v}$. That is, $\sigma(F)$ has an arc $(u, v)$ for each pair from $S_{u} \times T_{v}$ that is contained in $F$. This model was considered by Arratia et al. in [1, Section 7], who obtained an estimate of the expected number of Euler tours of a random $G \in \mathcal{G}_{\text {n }}^{\text {d,d }}$ for the case $d=2$. One nice property of the model, and of the original configuration model, is that directed graphs (without loops or double arcs) are generated with equal probability. Hence, by studying properties of uniformly random configurations it is possible to infer results about uniformly random elements of $\mathcal{G}_{\mathrm{n}}^{\mathrm{d}}$, by conditioning on there being no loops or double arcs.

In Section 2, we consider the configuration model for general (bounded) degree sequences. We first prove the useful combinatorial Lemma 2, which enumerates the number of partial configurations which map to in-directed forests with root set $R$. After that, in Theorem 3, we derive and prove exact expressions for the first and second moments for the number of arborescences of $\sigma(F)$, when $F$ is a configuration drawn uniformly at random 
from $\Phi_{n}^{\mathbf{d}}$. Next, in Theorem 5, we condition on the event that $\sigma(F)$ is a simple graph, to derive close approximations for the first and second moment, for the number of arborescences, when $G$ is a simple graph drawn uniformly at random from $\mathcal{G}_{\mathrm{n}}^{\mathbf{d}}$. As an immediate corollary we obtain corresponding approximations for the first and second moment when the random variable is the number of Euler tours. The expected value for the number of Euler tours over $\mathcal{G}_{\mathrm{n}}^{\mathbf{d}}$ is shown in Corollary 6 to tend to the value $\frac{e}{m}\left(\prod_{v \in[n]} d_{v}\right.$ !), which is a $\frac{e}{m}$ fraction of $|T S(G)|$. This allows us to infer that point (a), mentioned towards the end of Subsection 1.2, does hold.

In the analysis of random structures, it is sometimes the case that we can prove concentration (of a random variable within a fixed range) by applying Chebyshev's inequality to the first and second moment of that random variable. In the final part of Section 2 we show that the values of the first and second moments for Euler Tours in $\mathcal{G}_{\mathrm{n}}^{\mathrm{d}}$ are not good enough to prove concentration of measure using Chebyshev's inequality.

It is for the above reason that in Section 3 we use a more complicated method to show that the number of Euler tours for $G \in \mathcal{G}_{\mathrm{n}}^{\mathbf{d}}$ is asymptotically almost surely close to its expectation. The proof idea we use to obtain an asymptotic distribution is that of conditioning on short cycle counts, pioneered by Robinson and Wormald [12, 13]. Implicit in this pair of papers (and the subsequent work of Frieze et al. [5]) is a characterisation of the asymptotic distribution of the number of Hamiltonian cycles in a random $d$-regular graph in terms of random variables counting the number of $i$-cycles, for all fixed positive integers $i$. Janson [6] streamlined the technique of Robinson and Wormald and proved a general theorem (stated by us as Theorem 7). In Section 4, we use Theorem 7 to obtain an asymptotic distribution for the number of Euler tours of a random $d$-in $/ d$-out graph.

\section{Expectation and Variance of Euler tours}

In this section, we obtain the expectation and variance of the number of Euler tours of a random $G$ drawn from $\mathcal{G}_{\mathrm{n}}^{\mathrm{d}}$. In Section 3 we will go on to obtain the approximate asymptotic distribution of ETs in $d$-in/ $d$-out graphs.

We will use two particular facts several times in the proofs of this section. Recall the definition of falling factorial powers: for every $n, k \in \mathbb{N}$,

$$
(n)_{k}=n(n-1)(n-2) \cdots(n-k+1) .
$$

Fact 1. Falling factorial powers of sums obey the well known multinomial theorem

$$
\left(x_{1}+x_{2}+\cdots+x_{l}\right)_{k}=\sum_{\sum \delta_{i}=k}\left(\begin{array}{c}
k \\
\delta_{1}, \ldots, \delta_{l}
\end{array}\right) \prod_{i=1}^{l}\left(x_{i}\right)_{\delta_{i}},
$$

where the sum is taken over all partitions of $k$ into $l$ non-negative integer parts.

We have previously given the definition of a forest in Subsection 1.1. We will say that a forest $F$ is a $k$-forest if it is composed of exactly $k$ trees. The following fact will be used many times in this section of the paper: 
Fact 2 (see, e.g., [15](Theorem 5.3.4)). Let $V=\{1,2, \ldots, n\}$, and let $\delta=\left\{\delta_{v}: v \in V\right\}$ be a given vector of non-negative integers. The number of $k$-forests on $V$ in which $v$ has $\delta_{v}$ children is

$$
\left(\begin{array}{c}
n-1 \\
k-1
\end{array}\right)\left(\begin{array}{c}
n-k \\
\delta_{v}: v \in V
\end{array}\right)
$$

We use Fact 1 and Fact 2 to prove the following lemma. In this lemma, and in the proofs of subsequent results, we will speak of a configuration for an (in-directed) arborescence or forest. We take this to mean a partial matching from $S$ to $T$ (in the configuration model) that projects to an arborescence or a forest.

Lemma 2. Suppose we have a set of vertices $V=[n]$ for which there are $x_{v}$ points for arcs entering $v \in V$ and $y_{v}$ points for arcs leaving $v \in V$, with $x_{v}$ not necessarily equal to $y_{v}$. Assume $\sum_{v \in V} x_{v}>0$. Then the number of ways to choose a configuration for an in-directed forest rooted at $R \subseteq V$ is

$$
\left(\prod_{v \in V \backslash R} y_{v}\right)\left(\sum_{v \in R} x_{v}\right)\left(\sum_{v \in V} x_{v}-1\right)_{n-|R|-1} .
$$

Note that when $\sum_{v \in V} x_{v}=0$, there is only 1 forest possible, the forest consisting of $n$ isolated vertices (in this case we must have $R=V$ ).

Proof. First observe that if $R=[n]$, there is exactly 1 partial configuration which maps to a forest rooted at $R$. If we have $R \subset[n], R \neq[n]$ and also have $\sum_{v \in V} x_{v}=0$, there are 0 partial configurations mapping to a forest rooted at $R$.

From now on assume $R \neq[n]$ and $\sum_{v \in V} x_{v}=0$.

Consider some hypothetical (in-directed) forest $\mathcal{F}$ on $[n]$ rooted at $R$ and let $\delta_{v}$ be the number of children of $v$ in $\mathcal{F}$, for each $v \in V$ (Observe that we must have $\sum_{v \in V} \delta_{v}=$ $n-|R|)$. The number of ways to choose points for the source and target vertex of each $\operatorname{arc}$ in $\mathcal{F}$ is

$$
\left(\prod_{v \in V \backslash R} y_{v}\right)\left(\prod_{v \in V}\left(x_{v}\right)_{\delta_{v}}\right),
$$

since we must choose a point for the start of the arc directed away from each $v \notin R$ and choose one of the $x_{v}$ points for the end of each of the $\delta_{v} \operatorname{arcs}$ directed towards each $v \in V$.

Let $k=\sum_{v \in R} \delta_{v}$.

If $k=0$, then no vertex of $R$ has any incoming arcs. The only possible forest is the forest containing no arcs, which is not acceptable for the case $R \neq[n]$. Hence we need only consider the cases $k \geqslant 1$. Observe that for these cases, the task of constructing a forest rooted at $R$ and satisfying the child vector $\delta_{v}: v \in R$, is in one-to-one correspondence with first choosing any $k$-forest on $V \backslash R$, and then attaching each root of this forest as a child of some $v \in R$. Note the reason we will enumerate the forests in this way is to allow 
us to use Fact 2, which is not explicitly set up to allow us to specify particular roots. By Fact 2, the number of $k$-forests on $V \backslash R$ in which $v \in V \backslash R$ has exactly $\delta_{v}$ children is

$$
\left(\begin{array}{c}
n-|R|-1 \\
k-1
\end{array}\right)\left(\begin{array}{c}
n-|R|-k \\
\delta_{v}: v \in V \backslash R
\end{array}\right),
$$

and the number of ways to divide the roots of this forest amongst the members of $R$ so that each $v \in R$ has $\delta_{v}$ children is

$$
\left(\begin{array}{c}
k \\
\delta_{v}: v \in R
\end{array}\right)
$$

Now, to count all possible configurations for forests rooted at $R(R \neq[n])$, we consider all $k, 1 \leqslant k \leqslant n-|R|$, all possible vectors $\boldsymbol{\delta}$, and then combine (3), (4) and (5) to obtain

$$
\begin{aligned}
\left(\prod_{v \in V \backslash R} y_{v}\right) \times \sum_{k=1}^{n-|R|} & \left(\begin{array}{c}
n-|R|-1 \\
k-1
\end{array}\right)\left(\sum_{\left(\sum_{v \in R} \delta_{v}\right)=k}\left(\begin{array}{c}
k \\
\delta_{v}: v \in R
\end{array}\right) \prod_{v \in R}\left(x_{v}\right)_{\delta_{v}}\right) \\
& \times\left(\begin{array}{c}
n-|R|-k \\
\sum_{\left(\sum_{v \in V \backslash R} \delta_{v}\right)=n-|R|-k}\left(\begin{array}{c}
n-k \\
\delta_{v}: v \in V \backslash R
\end{array}\right) \prod_{v \in V \backslash R}\left(x_{v}\right)_{\delta_{v}}
\end{array}\right) .
\end{aligned}
$$

By Fact 1, we see that the two sums over the different $\delta_{v}$ in (6) are expansions of the falling factorial powers $\left(\sum_{v \in R} x_{v}\right)_{k}$ and $\left(\sum_{v \in V \backslash R} x_{v}\right)_{n-|R|-k}$, respectively. Hence, (6) is equal to

$$
\left(\prod_{v \in V \backslash R} y_{v}\right) \sum_{k=1}^{n-|R|}\left(\begin{array}{c}
n-|R|-1 \\
k-1
\end{array}\right)\left(\sum_{v \in R} x_{v}\right)_{k}\left(\sum_{v \in V \backslash R} x_{v}\right)_{n-|R|-k} .
$$

Applying Fact 1 again gives (2).

We now use Lemma 2 to analyse the expectation and variance of the number of arborescences in $\sigma(F)$, when $F$ is chosen uniformly at random from $\Phi_{n}^{\mathbf{d}}$. We say $\mathcal{A} \subset F$ is an arborescence of $F \in \Phi_{n}^{\mathbf{d}}$ if $\sigma(\mathcal{A})$ is an arborescence of $\sigma(F)$. In the following proofs, we will abuse terminology slightly and switch between speaking of arborescences of configurations and directed graphs arbitrarily. We will define $A R B S(F)$, for any $F \in \mathcal{G}_{\mathrm{n}}^{\mathrm{d}}$, to be the set of partial matchings on $S \times T$ which project to an Arborescence on $[n]$.

Theorem 3. Let $\mathbf{d}=\left(d_{1}, d_{2}, \ldots\right)$ be a sequence of positive integers. For each $n \in \mathbb{N}$, let $\mathcal{A}_{n}^{\star}$ denote the number of arborescences (rooted at any vertex) of a uniformly random $F \in \Phi_{n}^{\mathbf{d}}$. Then,

$$
\begin{aligned}
\mathbb{E}\left[\mathcal{A}_{n}^{\star}\right] & =\frac{n}{m}\left[\prod_{v \in[n]} d_{v}\right] ; \\
\mathbb{E}\left[\left(\mathcal{A}_{n}^{\star}\right)^{2}\right] & =\frac{m}{m-n+1} \mathbb{E}\left[\mathcal{A}_{n}^{\star}\right]^{2} .
\end{aligned}
$$


Proof. We start by computing the first moment of $\mathcal{A}_{n}^{\star}$. To calculate the first moment of $\mathcal{A}_{n}^{\star}$ we need to count the number of elements in the set

$$
\overline{\Phi_{n}^{\mathbf{d}}}=\left\{(F, \mathcal{A}): F \in \Phi_{n}^{\mathbf{d}}, \mathcal{A} \in A R B S(F)\right\}
$$

and then divide this quantity by $\left|\Phi_{n}^{\mathbf{d}}\right|$. Given $\mathcal{A}$, it is easy to count the number of configurations $F \in \Phi_{n}^{\mathbf{d}}$ for which $\mathcal{A} \subset F$. In any directed graph $G$ with $m$ arcs, there are exactly $m-n+1$ arcs not contained in any particular element of $A R B S(G)$. Hence, if we have a configuration for an arborescence, there are $(m-n+1)$ ! ways to extend this to a complete configuration. Applying Lemma 2 with $\mathbf{x}=\mathbf{y}=\mathbf{d}$, we see that the number of arborescences rooted at any particular vertex $v$ is

$$
d_{v}\left(\prod_{u \in[n] \backslash\{v\}} d_{u}\right)(m-1)_{n-2} .
$$

By the BEST theorem (Theorem 1), there are an equal number of arborescences rooted at each vertex of any $F \in \Phi_{n}^{\mathbf{d}}$. Hence, multiplying (8) by $n(m-n+1)$ ! gives

$$
\left|\overline{\Phi_{n}^{\mathbf{d}}}\right|=n(m-1) !\left(\prod_{v \in[n]} d_{v}\right) .
$$

Finally, dividing by the total number of configurations in $\Phi_{n}^{\mathbf{d}}$, which is $m$ !, gives the claimed value for $\mathbb{E}\left[\mathcal{A}_{n}^{\star}\right]$.

Next we evaluate $\mathbb{E}\left[\left(\mathcal{A}_{n}^{\star}\right)^{2}\right]$. To compute the second moment of $\mathcal{A}_{n}^{\star}$ we need to evaluate the following expression

$$
\frac{1}{m !} \sum_{F \in \Phi_{n}^{\mathbf{d}}}|A R B S(F)|^{2} .
$$

We observe that for any particular $F \in \Phi_{n}^{\mathbf{d}}$ the term $|A R B S(F)|^{2}$ in (10) is equal to the number of elements in the set

$$
\left\{\left(\mathcal{A}, \mathcal{A}^{\prime}\right): \mathcal{A}, \mathcal{A}^{\prime} \in A R B S(F)\right\}
$$

Hence

$$
\mathbb{E}\left[\left(\mathcal{A}_{n}^{\star}\right)^{2}\right]=\frac{\left|\widetilde{\Phi_{n}^{\mathbf{d}}}\right|}{\left|\Phi_{n}^{\mathbf{d}}\right|}
$$

where

$$
\widetilde{\Phi_{n}^{\mathbf{d}}}=\left\{\left(F, \mathcal{A}, \mathcal{A}^{\prime}\right): F \in \Phi_{n}^{\mathbf{d}}, \mathcal{A}, \mathcal{A}^{\prime} \in A R B S(F)\right\} .
$$

Hence, evaluating $\mathbb{E}\left[\left(\mathcal{A}_{n}^{\star}\right)^{2}\right]$ is equivalent to counting the elements of $\widetilde{\Phi_{n}^{\mathbf{d}}}$.

We compute $\left|\widetilde{\Phi_{n}^{\mathbf{d}}}\right|$ as follows. First, we count the number of ways to choose the intersection of a pair of arborescences $\mathcal{A}$ and $\mathcal{A}^{\prime}$. Then, we count the number of ways to extend this intersection to $\mathcal{A}$ and $\mathcal{A}^{\prime}$. Finally, we count the number of ways to choose the remainder of $F$ so that $\mathcal{A}$ and $\mathcal{A}^{\prime}$ are both in $A R B S(F)$. 
We start by considering the final stage. Suppose we have a partial configuration corresponding to a pair of arborescences $\left(\mathcal{A}, \mathcal{A}^{\prime}\right)$ and suppose $\mathcal{F}=\mathcal{A} \cap \mathcal{A}^{\prime}$ is a forest rooted at $R \subseteq[n]$. Since we need to add $|R|-1$ arcs to $\mathcal{F}$ to complete each arborescence, there must be $n+|R|-2$ edges in $\mathcal{A} \cup \mathcal{A}^{\prime}$. Hence, there are $(m-n-|R|+2)$ ! ways to choose the remaining edges for a configuration $F \in \Phi_{n}^{\mathrm{d}}$ which contains both $\mathcal{A}$ and $\mathcal{A}^{\prime}$.

Now we examine, for an arbitrary $R \subseteq[n]$, the number of different pairs $\left(\mathcal{A}, \mathcal{A}^{\prime}\right)$ with $\mathcal{F}=\mathcal{A} \cap \mathcal{A}^{\prime}$ rooted at $R$. In the analysis that follows, we will start by computing a weighted sum, with the weight of the pair of arborescences $\left(\mathcal{A}, \mathcal{A}^{\prime}\right)$ depending on the roots of $\mathcal{A}$ and $\mathcal{A}^{\prime}$. We use the BEST Theorem (Theorem 1) to get back to the correct number at the end of the proof.

We start by counting the number of ways we can choose $\mathcal{F}$, the edges in both arborescences, and then count the number of ways to choose the edges which are in one or the other arborescence. By Lemma 2 , if $R=[n]$ there is just 1 way to choose $\mathcal{F}$ rooted at $[n]$, but for $R \neq[n]$, the number of ways to choose $\mathcal{F}$ rooted at $R$ is

$$
\left(\prod_{v \in[n] \backslash R} d_{v}\right)\left(\sum_{v \in R} d_{v}\right)(m-1)_{n-|R|-1} .
$$

For each $v \in R$, let $\mathcal{F}_{v}$ denote the component of $\mathcal{F}$ with root $v$, and let $x_{v}$ be the number of points in $\bigcup_{u \in \mathcal{F}_{v}} T_{u}$ not used by arcs in $\mathcal{F}$ (recall from Subsection 1.3 that $T_{u}$ is the number of points originally available for arcs incoming to vertex $u$ ). That is,

$$
x_{v}=\sum_{u \in \mathcal{F}_{v}} d_{u}-\left|\mathcal{F}_{v}\right|+1
$$

Note that this is the number of points available to add arcs directed towards vertices of $\mathcal{F}_{v}$ when we are completing $\mathcal{A}$ and $\mathcal{A}^{\prime}$. Moreover, we have

$$
\sum_{v \in R} x_{v}=m-n+|R|
$$

We now turn our attention to the number of ways to choose $\mathcal{A} \backslash \mathcal{A}^{\prime}$ and $\mathcal{A}^{\prime} \backslash \mathcal{A}$. First note that if $|R|=1$ there is exactly one way to do this. Alternatively, for $|R| \geqslant 2$, choosing the remaining arcs for $\mathcal{A}$ and $\mathcal{A}^{\prime}$ is equivalent to choosing a pair of disjoint configurations for trees on $R$ in which there are $x_{v}$ points available for the targets of arcs entering $v$ and $d_{v}$ points available for the sources of the arcs leaving $v$, for each $v \in R$.

Suppose we have already chosen $\mathcal{A} \backslash \mathcal{A}^{\prime}$ such that the root of $\mathcal{A}$ is $r$ and suppose that there are $\delta_{v}$ arcs from $\mathcal{A} \backslash \mathcal{A}^{\prime}$ directed towards vertices in $\mathcal{F}_{v}$, for each $v \in R$. All the arcs of $\mathcal{A} \backslash \mathcal{A}^{\prime}$ must belong to the shared configuration $F$ which will contain $\mathcal{A}$ and $\mathcal{A}^{\prime}$. Hence for choosing $\mathcal{A}^{\prime} \backslash \mathcal{A}$, we have only $x_{v}-\delta_{v}$ points available for incoming arcs to $\mathcal{F}_{v}$, for $v \in R$. For outgoing arcs, we have $d_{v}-1$ points available for the source if $v \in R \backslash\{r\}$, or $d_{r}$ points available for the source of an arc leaving $r$.

First suppose we want to choose the tree $\mathcal{A}^{\prime} \backslash \mathcal{A}$ such that the root of $\mathcal{A}^{\prime}$ is $r^{\prime}$, where $r^{\prime} \neq r$. By Lemma 2 , the number of ways to choose $\mathcal{A}^{\prime} \backslash \mathcal{A}$, conditional on $\mathcal{A} \backslash \mathcal{A}^{\prime}$ having 
the child vector $\boldsymbol{\delta}$, is

$$
\left(x_{r^{\prime}}-\delta_{r^{\prime}}\right) d_{r}\left(\prod_{v \in R \backslash\left\{r, r^{\prime}\right\}}\left(d_{v}-1\right)\right)(m-n)_{|R|-2} .
$$

Now suppose both $\mathcal{A}$ and $\mathcal{A}^{\prime}$ are rooted at the same vertex $r \in R$. By Lemma 2, the number of ways to choose $\mathcal{A}^{\prime} \backslash \mathcal{A}$, conditional on $\mathcal{A} \backslash \mathcal{A}^{\prime}$ having the child vector $\boldsymbol{\delta}$, is

$$
\left(x_{r}-\delta_{r}\right)\left(\prod_{v \in R \backslash\{r\}}\left(d_{v}-1\right)\right)(m-n)_{|R|-2} .
$$

We now show how to combine (13) q (14)

We multiply $(13)$ by $\left(d_{r}-1\right)\left(d_{r^{\prime}}-1\right)$ and multiply (14) by $d_{r}\left(d_{r}-1\right)$. Then we sum over all choices for the root $r^{\prime}$ of $\mathcal{A}^{\prime}$ (but keep the root $r$ of $\mathcal{A}$ fixed) to get the following expression for the weighted sum of all configurations for $\mathcal{A}^{\prime} \backslash \mathcal{A}$, conditional on $\mathcal{A} \backslash \mathcal{A}^{\prime}$ having root $r$ :

$$
d_{r}\left(\prod_{v \in R}\left(d_{v}-1\right)\right)(m-n+1)_{|R|-1} .
$$

To derive the expression (15), we used the value for $\sum_{v \in R} x_{v}$ from the previous page, plus the fact that $\sum_{v \in R} \delta_{v}=|R|-1$. Note that (15) now is equal to a weighted sum over all arborescences $\mathcal{A}^{\prime} \backslash \mathcal{A}$ with any possible root $r^{\prime} \in R$, conditioned on the assumption that $\mathcal{A} \backslash \mathcal{A}^{\prime}$ has root $r$, in which $\mathcal{A}^{\prime}$ is weighted by a factor of $\left(d_{r}-1\right)\left(d_{r^{\prime}}-1\right)$ for $r^{\prime} \neq r$ and by a factor of $d_{r}\left(d_{r}-1\right)$ for $r^{\prime}=r$. We will correct to obtain the number of unweighted triples at the end of the proof.

Next, we must consider the number of ways to choose $\mathcal{A} \backslash \mathcal{A}^{\prime}$ with child vector $\boldsymbol{\delta}$ and with root $r$. For this step it is helpful to observe that no $\delta_{v}$ term appears in the overall value (15), obtained by summing over the weighted counts of numbers of ways to choose $\mathcal{A} \backslash \mathcal{A}^{\prime}$. Hence in considering the number of $\mathcal{A} \backslash \mathcal{A}^{\prime}$ configurations into root $r$, we can ignore the particular vector $\boldsymbol{\delta}$, and simply count all arborescences $\mathcal{A} \backslash \mathcal{A}^{\prime}$ on $R$ which have root $r$. Applying Lemma 2, the number of such configurations is

$$
\frac{x_{r}}{d_{r}}\left(\prod_{v \in R} d_{v}\right)(m-n+|R|-1)_{|R|-2} .
$$

Multiplying (15) by (16) gives the number of (weighted) configurations for $\left(\mathcal{A} \backslash \mathcal{A}^{\prime}, \mathcal{A}^{\prime} \backslash \mathcal{A}\right)$ when $\mathcal{A}$ has root $r$. Then summing over all choices for $r$ gives

$$
\left(\prod_{v \in R} d_{v}\right)\left(\prod_{v \in R}\left(d_{v}-1\right)\right)(m-n+|R|)_{2|R|-2}
$$

Multiplying by the number of ways to choose $\mathcal{F}$, given in (12), and the number of ways to choose the portion of $F$ not contained in $\mathcal{A} \cup \mathcal{A}^{\prime}$, which is $(m-n-|R|+2)$ !, yields 
the following expression

$$
\left(\sum_{v \in R} d_{v}\right)\left(\prod_{v \in V} d_{v}\right)\left(\prod_{v \in R}\left(d_{v}-1\right)\right)(m-1) ! .
$$

The expression (18) gives a weighted sum over triples $\left(F, \mathcal{A}, \mathcal{A}^{\prime}\right)$ in which the intersection $\mathcal{A} \cap \mathcal{A}^{\prime}$ is a forest rooted at $R$, for $|R|>1$. Each triple $\left(F, \mathcal{A}, \mathcal{A}^{\prime}\right)$ in which $\mathcal{A}$ and $\mathcal{A}^{\prime}$ are rooted at different vertices $u$ and $v$ is counted $\left(d_{u}-1\right)\left(d_{v}-1\right)$ times, and each triple $\left(F, \mathcal{A}, \mathcal{A}^{\prime}\right)$ in which $\mathcal{A}$ and $\mathcal{A}^{\prime}$ are rooted at the same vertex $v$ is counted $d_{v}\left(d_{v}-1\right)$ times. We also observe, that considering any $R \subseteq V$ such that $|R|=1$, that the number of triples $\left(F, \mathcal{A}, \mathcal{A}^{\prime}\right)$ is is exactly the number of pairs $(F, \mathcal{A})$ (since we must have $\mathcal{A}=\mathcal{A}^{\prime}$ in this case). Applying Lemma 2 with $x_{v}=y_{v}=d_{v}$, multiplying by the number $(m-n+1)$ ! of ways of completing the configuration, and then multiplying by $d_{r}\left(d_{r}-1\right)$ (in order to achieve the appropriate weight for this case), we obtain the exact value of (18) for this $R$. Hence (18) can be used for the $|R|=1$ case also.

Only the second two factors of (18) depend on $R$. Summing these over all $R \subseteq V$ gives

$$
\sum_{R \subseteq V}\left(\sum_{v \in R} d_{v}\right)\left(\prod_{v \in R}\left(d_{v}-1\right)\right)
$$

We can evaluate (19) by separating it into $n$ separate sums, each corresponding to the sum over $R \ni v$ for a particular $v \in[n]$,

$$
d_{v} \sum_{R \ni v} \prod_{u \in R}\left(d_{u}-1\right)=\left(d_{v}-1\right)\left(\prod_{u \in V} d_{u}\right) .
$$

Summing the right-hand side of (20) over each $v \in V$ and combining with the rest of (18) gives

$$
\left(\prod_{v \in V} d_{v}\right)^{2}(m-n)(m-1) !
$$

We cannot immediately obtain the quantity we are looking for from (21) as its different triples have been weighted by different amounts. However, by the BEST theorem (Theorem 1), we know that the number of triples $\left(F, \mathcal{A}, \mathcal{A}^{\prime}\right)$ in which $\mathcal{A}$ is rooted at $u$ and $\mathcal{A}^{\prime}$ is rooted at $v$ does not depend on $u$ or $v$, since the projection $\sigma(F)$ is always an Eulerian directed graph. Thus, it follows that the factor by which (21) over-counts the number of triples is

$$
\frac{1}{n^{2}}\left(\sum_{u \neq v}\left(d_{u}-1\right)\left(d_{v}-1\right)+\sum_{v} d_{v}\left(d_{v}-1\right)\right)=\frac{(m-n+1)(m-n)}{n^{2}} .
$$

Dividing (21) by (22) gives

$$
\left|\widetilde{\Phi_{n}^{\mathbf{d}}}\right|=\frac{n^{2}}{m-n+1}\left(\prod_{v \in V} d_{v}\right)^{2}(m-1) !
$$


Finally, dividing $\left|\widetilde{\Phi_{n}^{\mathbf{d}}}\right|$ by $m$ ! gives

$$
\mathbb{E}\left[\left(\mathcal{A}_{n}^{\star}\right)^{2}\right]=\frac{n^{2}}{m(m-n+1)}\left(\prod_{v \in V} d_{v}\right)^{2} .
$$

Recall that simple directed graphs are generated with equal probability in the configuration model. Thus, by conditioning on $\sigma(F)$ containing no loops or 2-cycles, we can obtain the first two moments of the number of arborescences of a uniformly random $G \in \mathcal{G}_{\mathrm{n}}^{\mathrm{d}}$. Before we show this, in Theorem 5, we prove a useful lemma regarding small subgraphs, which will also be used in Section 3.

Lemma 4. Let $r$ be some fixed positive integer and let $F$ be chosen uniformly at random from $\Phi_{n}^{\mathbf{d}}$. The probability that $\sigma(F)$ contains any set of $r$ vertices that induce a subgraph with more arcs than vertices tends to 0 as $n \rightarrow \infty$.

The claim also holds when $F$ is obtained as the first part of a uniformly random $(F, \mathcal{A}) \in \overline{\Phi_{n}^{\mathbf{d}}}$ (defined in $(7)$ above), or when $F$ is obtained as the first part of a uniformly random $\left(F, \mathcal{A}, \mathcal{A}^{\prime}\right) \in \widetilde{\Phi_{n}^{\mathbf{d}}}$ (defined in (11) above).

Proof. Let $q$ be a probability distribution on $\Phi_{n}^{\mathbf{d}}$ and let $F^{\prime}$ be a set of $k$ distinct configuration edges, for some fixed positive integer $k$. We will show that the claim holds whenever $q$ satisfies

$$
\sum_{F \supseteq F^{\prime}} q(F) \in O\left(m^{-k}\right)
$$

for any choice of $F^{\prime}$, and then show that the three distributions in question all satisfy (24).

Suppose we have a directed graph $H$ with $r$ vertices and $r+s$ arcs, where $r$ and $s$ are fixed positive integers. The number of ways to choose a partial configuration $F^{\prime}$ with $\sigma\left(F^{\prime}\right)$ isomorphic to $H$ is $O\left(n^{r}\right)$ - there are $\left(\begin{array}{l}n \\ r\end{array}\right)$ ways to choose the vertices, and the $d$-bound on degree of vertices means there are only a constant (depending on $d, r+s$ ) number of ways to configure the arcs. Moreover, the number of different graphs on $r$ vertices with $r+s$ arcs only depends on $r$ and $s$, so the total number of partial configurations which project to any such $H$ is also $O\left(n^{r}\right)$. Hence, when $F$ is chosen according to $q$ satisfying (24), the probability that $\sigma(F)$ contains any $r$-set of vertices which induce a subgraph with $r+s$ edges is $O\left(n^{-s}\right)$. Observe that for a fixed $r$ there are at most $r^{2}-r$ possible values for $s$, so the probability that we have a subgraph with $r$ vertices and more than $r$ arcs is $O\left(n^{-1}\right)$.

Suppose we have a partial configuration $F^{\prime}$ of size $k$, for some fixed positive integer $k$. The number of ways to extend $F^{\prime}$ to a full configuration $F \in \Phi_{n}^{\mathbf{d}}$ is equal to $\left|\Phi_{n}^{\mathbf{d}^{\prime}}\right|$, where $\mathbf{d}^{\prime}$ gives the remaining in/out-degrees of vertices once the points used in $F^{\prime}$ have been removed. Hence, the probability that $F^{\prime}$ is contained in a randomly chosen configuration $F \in \Phi_{n}^{\mathbf{d}}$ is equal to

$$
\left|\Phi_{n}^{\mathbf{d}^{\prime}}\right| /\left|\Phi_{n}^{\mathbf{d}}\right|=1 /(m)_{k} \in O\left(m^{-k}\right) .
$$

Similarly, when $F$ is obtained as the first part of a uniformly random element $(F, \mathcal{A}) \in$ $\overline{\Phi_{n}^{\mathbf{d}}}$ or, respectively, a uniformly random element $\left(F, \mathcal{A}, \mathcal{A}^{\prime}\right) \in \widetilde{\Phi_{n}^{\mathbf{d}}}$, we can see that the lefthand side of (24) is at most $\left|\overline{\Phi_{n}^{\mathbf{d}^{\prime}}}\right| /\left|\overline{\Phi_{n}^{\mathbf{d}}}\right|$ (resp. $\left|\widetilde{\Phi_{n}^{\mathbf{d}^{\prime}}}\right| /\left|\widetilde{\Phi_{n}^{\mathbf{d}}}\right|$ ). By (9) and (23), both these quantities are $O\left(m^{-k}\right)$. 
Theorem 5. Let $d$ be some fixed constant, let $\mathbf{d}=\left(d_{1}, d_{2}, \ldots\right)$ be a sequence of positive integers satisfying $d_{i} \leqslant d$ for all $i$, let $n \in \mathbb{N}$, and let $m=\sum_{v=1}^{n} d_{v}$. Assume that $V_{1}$, the set of vertices $u$ such that $d_{u}=1$, satisfies the condition $\left|[n] \backslash V_{1}\right|=\Omega(n)$ (observe this implies $m-n \rightarrow \infty)$. Let $\mathcal{A}_{n}$ denote the number of arborescences of a directed graph chosen randomly from $\mathcal{G}_{\mathrm{n}}^{\mathrm{d}}$. Then

$$
\begin{aligned}
& \mathbb{E}\left[\mathcal{A}_{n}\right] \sim e \frac{n}{m}\left[\prod_{v \in[n]} d_{v}\right] \\
& \mathbb{E}\left[\mathcal{A}_{n}^{2}\right] \sim e^{-n / m} \frac{m}{m-n} \mathbb{E}\left[\mathcal{A}_{n}\right]^{2} .
\end{aligned}
$$

Proof. In the following we will use $m_{2}$ to denote $\sum_{v} d_{v}^{2}$.

The proof is as follows. We say $F \in \Phi_{n}^{\mathrm{d}}$ contains a loop at $v$ if there is an edge from $S_{v} \times T_{v}$ in $F$ and that $F$ contains a double arc from $u$ to $v$ if there is a pair of edges from $S_{u} \times T_{v}$ in $F$. Let $L$ and $D$ denote the number of loops and double arcs in a random $F \in \Phi_{n}^{\mathrm{d}}$. Then, the event " $F$ is simple" is equivalent to the event $\{L=D=0\}$. We first analyse the distributions of $L$ and $D$, which we can use to estimate the probability that $F$ is simple. Then, we consider two new random variables, $L^{(1)}$ and $D^{(1)}$, which count the number of loops and double arcs in $F$ when $(F, \mathcal{A})$ is chosen randomly from the set $\overline{\Phi_{n}^{\mathrm{d}}}$, defined in (7). Hence, by analysing the distributions of $L^{(1)}$ and $D^{(1)}$, we will be able to estimate $\mathbb{E}\left[\mathcal{A}_{n}\right]$ using

$$
\mathbb{E}\left[\mathcal{A}_{n}\right]=\frac{\mathbb{P}\left[L^{(1)}=D^{(1)}=0\right]}{\mathbb{P}[L=D=0]} \mathbb{E}\left[\mathcal{A}_{n}^{\star}\right] .
$$

Finally, we consider random variables, $L^{(2)}$ and $D^{(2)}$, which count the number of loops and double arcs in $F$, when $\left(F, \mathcal{A}, \mathcal{A}^{\prime}\right)$ is chosen randomly from the set $\widetilde{\Phi_{n}^{\mathbf{d}}}$, defined in (11). Hence, by analysing the distributions of $L^{(2)}$ and $D^{(2)}$, we will be able to estimate $\mathbb{E}\left[\left(\mathcal{A}_{n}\right)^{2}\right]$ using

$$
\mathbb{E}\left[\left(\mathcal{A}_{n}\right)^{2}\right]=\frac{\mathbb{P}\left[L^{(2)}=D^{(2)}=0\right]}{\mathbb{P}[L=D=0]} \mathbb{E}\left[\left(\mathcal{A}_{n}^{\star}\right)^{2}\right] .
$$

We first compute the expectation of $L$ and $D$. Suppose we have a loop edge $e \in S_{v} \times T_{v}$ in $F$ and let $I_{e}$ be the indicator variable for the event $e \in F$. Then, we can write $L=\sum_{v \in V} \sum_{e \in S_{v} \times T_{v}} I_{e}$ and, by linearity of expectation, we have

$$
\mathbb{E}[L]=\sum_{v \in V} \sum_{e \in S_{v} \times T_{v}} \mathbb{E}\left[I_{e}\right]=\sum_{v \in V} \sum_{e \in S_{v} \times T_{v}} \mathbb{P}[e \in F] .
$$

Given $e$, the number of ways to choose $F$ with $e \in F$ is $(m-1)$ !, so the probability of a random $F \in \Phi_{n}^{\mathrm{d}}$ containing $e$ is $1 / m$. For each $v$, there are $d_{v}^{2}$ ways to choose an edge from $S_{v} \times T_{v}$. Hence,

$$
\mathbb{E}[L]=\frac{1}{m} \sum_{v} d_{v}^{2}=\frac{m_{2}}{m} .
$$

Observe this expression is $\Theta(1)$. 
Next, we compute the expectation of $D$. Here, for every pair of edges $e, f \in S_{u} \times T_{v}$, for some $u \neq v$, we define an indicator variable $I_{e, f}$ for the event $e, f \in F$. By linearity of expectation, we have

$$
\mathbb{E}[D]=\sum_{u \in V} \sum_{v \in V \backslash\{u\}} \sum_{e, f \in S_{u} \times T_{v}} \mathbb{P}[e, f \in F] .
$$

The probability of a particular pair of edges $e$ and $f$ occurring in a random configuration $F \in \Phi_{n}^{\mathbf{d}}$ is, asymptotically, $1 / \mathrm{m}^{2}$. Moreover, the number of ways to choose $e, f \in S_{u} \times T_{v}$ is $2\left(\begin{array}{c}d_{u} \\ 2\end{array}\right)\left(\begin{array}{c}d_{v} \\ 2\end{array}\right)$. Hence, the sum in $(27)$ becomes

$$
\begin{aligned}
\mathbb{E}[D] & \sim \frac{2}{m^{2}} \sum_{u \in V} \sum_{v \in V \backslash\{u\}}\left(\begin{array}{c}
d_{u} \\
2
\end{array}\right)\left(\begin{array}{c}
d_{v} \\
2
\end{array}\right) \\
& =\frac{1}{2 m^{2}}\left(\sum_{u \in V}\left(d_{u}\right)_{2}\right)^{2}-\frac{1}{2 m^{2}} \sum_{u \in V}\left(d_{u}\right)_{2}^{2} .
\end{aligned}
$$

To finish the calculation we observe that the negative term in $(28)$ is $O(1 / m)$ (each $d_{u}$ is bounded above by a constant $d$, so $\left.\sum_{u}\left(d_{u}\right)_{2}^{2} \leqslant d^{3} m\right)$. Hence, this part of the sum goes to 0 as $m \rightarrow \infty$ and we see that

$$
\mathbb{E}[D] \sim \frac{\left(m_{2}-m\right)^{2}}{2 m^{2}} .
$$

Note that $m_{2}-m=\sum_{v \in V} d_{v}\left(d_{v}-1\right)=\sum_{v \in V \backslash V_{1}} d_{v}\left(d_{v}-1\right) \geqslant 2\left|V \backslash V_{1}\right|$, using the fact that $d_{v}\left(d_{v}-1\right)=0$ for all $v \in V_{1}$ and $d_{v}\left(d_{v}-1\right) \geqslant 2$ for $v \in V \backslash V_{1}$. We now apply our assumption that $\left|V \backslash V_{1}\right| \geqslant c n$ in the limit (for the $c$ of the $\Omega(n)$ ) to observe that $m_{2}-m \geqslant 2 c n$. We also know $m \leqslant d n$ by the fact that degrees are bounded. Hence $\frac{m_{2}-m}{m} \geqslant \frac{2 c}{d}$ as $n \rightarrow \infty$, and hence $\mathbb{E}[D]$ tends to some value which is $\Theta(1)$.

We will now show that $L$ and $D$ converge to a pair of (asymptotically) independent Poisson random variables and, therefore, the probability that $F$ is simple when $F$ is chosen uniformly at random from $\Phi_{n}^{\mathbf{d}}$ satisfies

$$
\mathbb{P}[L=D=0] \sim \exp \left(-\frac{m_{2}}{m}-\frac{\left(m_{2}-m\right)^{2}}{2 m^{2}}\right) .
$$

To show that $L$ and $D$ converge to a pair of (asymptotically) independent Poisson random variables, we need to show that, for any pair of fixed positive integers $j$ and $k$,

$$
\mathbb{E}\left[(L)_{j}(D)_{k}\right] \sim \mathbb{E}[L]^{j} \mathbb{E}[D]^{k}
$$

$\mathbb{E}\left[(L)_{j}(D)_{k}\right]$ is computed as the expected number of ordered tuples of $j$ loops and $k$ double arcs in a uniformly random $F \in \Phi_{n}^{\mathbf{d}}$. By Lemma 4 , and by the fact that $\mathbb{E}[L]$ and $\mathbb{E}[D]$ are $\Omega(1)$, we can assume that the contribution to $\mathbb{E}\left[(L)_{j}(D)_{k}\right]$ from tuples of loops and double arcs with repeated vertices goes to 0 as $n \rightarrow \infty$. Hence, we can assume loops and double arcs occur independently; that is, (31) holds as $n \rightarrow \infty$. 
We remark that, since Lemma 4 holds for the case when $F$ is obtained as the first element of a uniformly random element of $\overline{\Phi_{n}^{\mathbf{d}}}$ (resp. when $F$ is obtained as the first element of a uniformly random element of $\left.\widetilde{\Phi_{n}^{\mathbf{d}}}\right)$, it will be possible to use similar arguments to those in the previous paragraph to show that the random variables $L^{(1)}$ and $D^{(1)}$ (resp. $L^{(2)}$ and $D^{(2)}$ ) converge to a pair of (asymptotically) independent Poisson random variables. We first compute the expectations of $L^{(1)}, D^{(1)}$ and of $L^{(2)}, D^{(2)}$.

Consider the distributions of $L^{(1)}$ and $D^{(1)}$. We first estimate $\mathbb{E}\left[L^{(1)}\right]$. Suppose we have a loop edge $e \in S_{v} \times T_{v}$, for some $v \in V$. A loop edge cannot be contained in any arborescence, and, thus, the number of pairs $(F, \mathcal{A}) \in \overline{\Phi_{n}^{\mathbf{d}}}$ where $e \in F$, is equal to the number of pairs $(F, \mathcal{A}) \in \overline{\Phi_{n}^{\mathbf{d}^{\prime}}}$, where $\mathbf{d}^{\prime}$ is equal to $\mathbf{d}$ with $d_{v}$ replaced by $d_{v}-1$. Hence, adapting the expression for $\left|\overline{\Phi_{n}^{\mathbf{d}}}\right|$ computed earlier in (9), we can see that the number of elements of $\overline{\Phi_{n}^{\mathbf{d}}}$ with $e \in F$ is equal to

$$
n\left(d_{v}-1\right) \prod_{u \in V \backslash\{v\}} d_{u}(m-2) !
$$

Dividing (32) by the total number of elements in $\overline{\Phi_{n}^{\mathbf{d}}}$ gives the probability

$$
\mathbb{P}\left[e \in F:(F, \mathcal{A}) \in \overline{\Phi_{n}^{\mathbf{d}}}\right]=\frac{d_{v}-1}{d_{v}(m-1)}
$$

Evaluating (25) with this probability in the place of $\mathbb{P}[e \in F]$ gives

$$
\mathbb{E}\left[L^{(1)}\right]=\frac{1}{m-1} \sum_{v \in V} d_{v}\left(d_{v}-1\right) \sim \frac{m_{2}-m}{m} .
$$

Recall from the work on $\mathbb{E}[D]$ that this limiting expression $\left(m_{2}-m\right) / m$ has some $\Theta(1)$ value.

Next, we evaluate $\mathbb{E}\left[D^{(1)}\right]$. Suppose we have a pair of edges $e, f \in S_{u} \times T_{v}$ for some $u \neq v$. By Lemma 2, the number of arborescences rooted at $u$ in which each $w \notin\{u, v\}$ has $d_{w}$ points available for its incoming and outgoing arcs, $u$ has $d_{u}$ points available for incoming arcs, and $v$ has $d_{v}-2$ points available for incoming arcs and $d_{v}$ available for outgoing arcs is

$$
\left(\prod_{w=1}^{n} d_{w}\right)(m-3)_{n-2} .
$$

The expression in (34) counts the number of partial configurations which consist of the edges $e$ and $f$ along with $n-1$ configuration edges that project to an arborescence rooted at $u$. There are $(m-n-1)$ ! ways to extend each of these partial configurations to some $F \in \Phi_{n}^{\mathbf{d}}$. Hence, the following expression counts the number of pairs $(F, \mathcal{A}) \in \overline{\Phi_{n}^{\mathbf{d}}}$ with $e, f \in F$ and $\mathcal{A}$ rooted at $u$ :

$$
\left(\prod_{w=1}^{n} d_{w}\right)(m-3) !
$$


By the BEST Theorem (Theorem 1), we know that each $F \in \Phi_{n}^{\mathrm{d}}$ has the same number of arborescences rooted at each vertex, so $(35)$ counts exactly $1 / n$ of the pairs $(F, \mathcal{A}) \in \overline{\Phi_{n}^{\mathrm{d}}}$ with $e, f \in F$. Multiplying (35) by $n$ and dividing by the value $\left|\overline{\Phi_{n}^{\mathbf{d}}}\right|$ given in (9) gives

$$
\mathbb{P}\left[e, f \in F:(F, \mathcal{A}) \in \overline{\Phi_{n}^{\mathbf{d}}}\right] \sim \frac{1}{m^{2}} .
$$

This is the same probability as when $F$ is chosen uniformly at random from $\Phi_{n}^{\mathrm{d}}$, so evaluating (29) with (36) in place of $\mathbb{P}[e, f \in F]$ does not change the (asymptotic) value, and we have

$$
\mathbb{E}\left[D^{(1)}\right] \sim \mathbb{E}[D] .
$$

Now that we have that shown $L^{(1)}$ and $D^{(1)}$ to be $\Omega(1)$, we can use Lemma 4 to infer that $L^{(1)}$ and $D^{(1)}$ converge to (asymptotically) independent Poisson random variables. Hence we can see that the probability of $F$ being simple in a random $(F, \mathcal{A}) \in \overline{\Phi_{n}^{\mathrm{d}}}$ satisfies

$$
\mathbb{P}\left[L^{(1)}=D^{(1)}=0\right] \sim \exp \left(-\frac{m_{2}-m}{m}-\frac{\left(m_{2}-m\right)^{2}}{2 m^{2}}\right) .
$$

Together (30) and (37) give the claimed estimate for $\mathbb{E}\left[\mathcal{A}_{n}\right]$.

Finally, we consider the distributions of $L^{(2)}$ and $D^{(2)}$. First, suppose we have a loop edge $e \in S_{v} \times T_{v}$. The number of elements of $\widetilde{\Phi_{n}^{\mathbf{d}}}$ with $e \in F$ is equal to the number of elements of $\widetilde{\Phi_{n}^{\mathrm{d}^{\prime}}}$, where $\mathbf{d}^{\prime}$ is the out-degree vector we used to compute $\mathbb{E}\left[L^{(1)}\right]$. Adapting the expression (23), we have

$$
\left|\widetilde{\Phi_{n}^{\mathrm{d}^{\prime}}}\right|=\frac{\left(d_{v}-1\right)^{2}}{\left(d_{v}\right)^{2}} \frac{n^{2}}{m-n}\left(\prod_{w=1}^{n} d_{w}\right)^{2}(m-2) ! .
$$

Dividing by the number of elements in $\widetilde{\Phi_{n}^{\mathbf{d}}}$ (explicitly given in (23)) we see that

$$
\mathbb{P}\left[e \in F:\left(F, \mathcal{A}, \mathcal{A}^{\prime}\right) \in \widetilde{\Phi_{n}^{\mathbf{d}}}\right] \sim \frac{\left(d_{v}-1\right)^{2}}{\left(d_{v}\right)^{2} m} .
$$

Evaluating (26) with this probability in the place of $\mathbb{P}[e \in F]$ gives

$$
\mathbb{E}\left[L^{(2)}\right] \sim \frac{m_{2}-2 m+n}{m},
$$

which is $\Theta(1)$ under our restriction on the number of $d_{u}=1$ vertices.

We now evaluate $\mathbb{E}\left[D^{(2)}\right]$. Suppose we have a pair of $\operatorname{arcs} e, f \in S_{u} \times T_{v}$ for some $u \neq v$. Observe that it must be the case that $d_{u} \geqslant 2, d_{v} \geqslant 2$; otherwise the scenario cannot arise. There are three cases to consider:

(i) when both $\mathcal{A}$ and $\mathcal{A}^{\prime}$ contain an arc from $\{e, f\}$;

(ii) when neither $\mathcal{A}$ nor $\mathcal{A}^{\prime}$ contain an arc from $\{e, f\}$; 
(iii) when exactly one of $\mathcal{A}, \mathcal{A}^{\prime}$ contains an arc from $\{e, f\}$.

Using slightly more general arguments than those used to compute the second moment in Theorem 3, we count the number of triples $\left(F, \mathcal{A}, \mathcal{A}^{\prime}\right)$ for each of these three cases, obtaining expressions which count weighted triples in the same way as (21). Then we will show that the factor by which we over-count triples is almost identical in each of the three cases above. We will be able to add the contributions of these three expressions together, apply the BEST theorem, and proceed as we did in the proof of Theorem 3.

In each of the three cases, we want to count pairs of arborescences using some subset of the configuration points. Suppose we are working with sets of points where $s_{w}=\left|S_{w}\right|$ and $t_{w}=\left|T_{w}\right|$ for each vertex $w$, with $s_{w}$ not necessarily equal to $t_{w}$, and with $\sum_{w \in V} s_{w} \leqslant$ $\sum_{w \in V} t_{w}$ and $s_{w} \geqslant 1, t_{w} \geqslant 1$ for all $w$. In this model, we will consider a configuration to be any maximal matching from $\bigcup_{w \in V} S_{w}$ to $\bigcup_{w \in V} T_{w}$. Note that the fact that the in-degree and out-degrees are equal is only used in the final step of the analysis of the second moment of $\mathcal{A}_{n}^{\star}$ (in Theorem 3). Thus, by following the arguments of the second part of Theorem 3 we find that, for each $R \subseteq V$, the expression giving a weighted sum over triples $\left(F, \mathcal{A}, \mathcal{A}^{\prime}\right)$ where $\mathcal{A} \cap \mathcal{A}^{\prime}$ is a forest rooted at $R$ (given by (18) in the proof of Theorem 3) becomes

$$
\left(\sum_{w \in R} t_{w}\right)\left(\prod_{w \in R}\left(s_{w}-1\right)\right)\left(\prod_{w \in V} s_{w}\right) \frac{\left(m_{t}-1\right) !}{\left(m_{t}-m_{s}\right) !},
$$

where $m_{t}=\sum_{w} t_{w}$ and $m_{s}=\sum_{w} s_{w}$. The $1 /\left(m_{t}-m_{s}\right)$ ! term in (39) comes from the fact that the number of ways to choose $F \backslash\left(\mathcal{A} \cup \mathcal{A}^{\prime}\right)$ is now

$$
\frac{\left(m_{t}-n-|R|+2\right) !}{\left(m_{t}-m_{s}\right) !} \text {. }
$$

The factor by which $(39)$ weights $\left(F, \mathcal{A}, \mathcal{A}^{\prime}\right)$ is $\left(s_{r}-1\right)\left(s_{r^{\prime}}-1\right)$ if $\mathcal{A}$ and $\mathcal{A}^{\prime}$ are rooted at different vertices $r, r^{\prime} \in R$, and is $s_{r}\left(s_{r}-1\right)$ if both are rooted at the same vertex $r \in R$. Summing (39) over all possibilities for $R$ gives

$$
\left(\sum_{w \in V} \frac{t_{w}\left(s_{w}-1\right)}{s_{w}}\right)\left(\prod_{w \in V} s_{w}\right)^{2} \frac{\left(m_{t}-1\right) !}{\left(m_{t}-m_{s}\right) !} .
$$

case (i): First, suppose both $\mathcal{A}$ and $\mathcal{A}^{\prime}$ contain an element from $\{e, f\}$. In this case, choosing $\mathcal{A}$ and $\mathcal{A}^{\prime}$ is equivalent to choosing a pair of arborescences in a configuration model where we have contracted $(u, v)$ to a single vertex, which we will name $v$. That is, we have a pair of degree vectors $\mathbf{s}$ and $\mathbf{t}$, each of length $n-1$, where $s_{v}=d_{v}, t_{v}=d_{u}+d_{v}-2$, and $s_{w}=t_{w}=d_{w}$ for $w \in V \backslash\{u, v\}$. Any maximal matching in this configuration model can be extended to a configuration $F \in \Phi_{n}^{\mathbf{d}}$ by matching the remaining $d_{u}-2$ outgoing points of $u$ (in any of $\left(d_{u}-2\right)$ ! ways) with the unallocated points from $T$. Thus, by directly applying (40) and then multiplying by $\left(d_{u}-2\right)$ ! the sum over weighted triples is

$$
\frac{1}{d_{u}^{2}}\left(m-n-1-\frac{d_{u}-2}{d_{v}}\right)\left(\prod_{w \in V} d_{w}\right)^{2}(m-3) !
$$


where we weight by a factor of $d_{r}\left(d_{r}-1\right)$ for arborescence pairs with the same root $r \in$ $V \backslash\{u\}$, and by a factor of $\left(d_{r}-1\right)\left(d_{r^{\prime}}-1\right)$ for arborescence pairs with roots $r, r^{\prime} \in V \backslash\{u\}$ respectively, $r \neq r^{\prime}$. There are 4 ways to choose an arc for each of $\mathcal{A}$ and $\mathcal{A}^{\prime}$ from the set $\{e, f\}$. Multiplying (41) by 4 , we see that as $m-n \rightarrow \infty$ (this is guaranteed by the restriction on the number of degree 1 vertices), the sum over weighted triples for which both $\mathcal{A}$ and $\mathcal{A}^{\prime}$ have an arc from $\{e, f\}$ is, asymptotically,

$$
(m-n) \frac{4}{\left(d_{u}\right)^{2}}\left(\prod_{w \in V} d_{w}\right)^{2}(m-3) !
$$

case (ii): Next, suppose neither $\mathcal{A}$ nor $\mathcal{A}^{\prime}$ contain an element from $\{e, f\}$. To count the number of triples of this form we first observe that if $d_{u}=2$, then for any $F$ containing $e, f$, the set of arborescences which contain neither $e$ nor $f$ are exactly the arborescences which have root $u$. By the BEST theorem, the number of triples $\left(F, \mathcal{A}, \mathcal{A}^{\prime}\right)$ in which $\mathcal{A}, \mathcal{A}^{\prime}$ both have root $u$, and $e, f$ both belong to $F$, is a $1 / n^{2}$ fraction of the total number of triples where $e, f \in F$, this total being the overall value we aim to compute. For now we observe that if $d_{u}=2$, the $e, f \notin \mathcal{A} \cup \mathcal{A}^{\prime}$ subcase contributes only a $n^{-2}$ fraction of this eventual number of triples.

Now assume $d_{u}>2$. We evaluate (40) on $V$ with $s_{w}=d_{w}$ for $w \neq u, s_{u}=d_{u}-2$, $t_{w}=d_{w}$ for $w \neq v$, and $t_{v}=d_{v}-2$, since we remove two points from each of $S_{u}$ and $T_{v}$. We have $m_{s}=m_{t}$ so, in this case, (40) evaluates to

$$
\left(m-n-\frac{d_{u}}{d_{u}-2}-\frac{d_{v}-2}{d_{v}}\right) \frac{\left(d_{u}-2\right)^{2}}{d_{u}^{2}}\left(\prod_{w \in V} d_{w}\right)^{2}(m-3) !,
$$

or, asymptotically, as $m-n \rightarrow \infty$ (implied by our restriction on $\left|V_{1}\right|$ ),

$$
(m-n) \frac{\left(d_{u}-2\right)^{2}}{d_{u}^{2}}\left(\prod_{w \in V} d_{w}\right)^{2}(m-3) !
$$

case (iii): Finally, suppose exactly one of $\mathcal{A}, \mathcal{A}^{\prime}$ contains an element of $\{e, f\}$.

First consider the case where $d_{u}=2$. Suppose $\mathcal{A}$ is the arborescence to contain the element of $\{e, f\}$. Then by $d_{u}=2$, we must have $\mathcal{A}^{\prime}$ rooted at $u$. By the BEST theorem, the proportion of arborescences $\mathcal{A}^{\prime}$ of $F$ rooted at $u$ for any Eulerian configuration $F$ is exactly a $1 / n$ fraction of all arborescences in $F$. Also, by $d_{u}=2$, the arborescences $\mathcal{A}$ containing one of $e, f$ for an Eulerian configuration $F, F \ni e, f$ are exactly those arborescences which are not rooted at $u$. Hence the number of such $\mathcal{A}$ arboresences is exactly an $(n-1) / n$ fraction of all arborescences in $F$. Multiplying by 2 to account for $\mathcal{A}, \mathcal{A}^{\prime}$ switching roles, the number of triples $\left(F, \mathcal{A}, \mathcal{A}^{\prime}\right)$ with $e, f \in F$ such that exactly one of $\mathcal{A}$ and $\mathcal{A}^{\prime}$ is rooted at $u$ is a $2(n-1) /\left(n^{2}\right)$ fraction of all number of triples $\left(F, \mathcal{A}, \mathcal{A}^{\prime}\right)$ where $e, f \in F$. This latter quantity is what we aim to eventually compute. For now we note that when $d_{u}=2$, the subcase of $|\mathcal{A} \cap\{e, f\}|+\left|\mathcal{A}^{\prime} \cap\{e, f\}\right|=1$ only contributes a $2(n-1) / n^{2}$ fraction of all triples. 
Alternatively, we have $d_{u}>2$. We will count triples $\left(F, \mathcal{A}, \mathcal{A}^{\prime}\right)$ where $e, f \in F, e \in$ $\mathcal{A}, e \notin \mathcal{A}^{\prime}, f \notin \mathcal{A} \cup \mathcal{A}^{\prime}$. The argument is similar to that of Theorem 3. We first count the number of completions of a pair $\left(\mathcal{A}, \mathcal{A}^{\prime}\right)$ to a configuration $F$. If $\mathcal{A} \cap \mathcal{A}^{\prime}$ has $\rho$ components, the number of arcs already in $\mathcal{A} \cup \mathcal{A}^{\prime}$ (which includes $e$ ) is $n+\rho-2$. However $f$, which is not in $\mathcal{A} \cup \mathcal{A}^{\prime}$, is also committed to $F$. Hence there are $(m-n-\rho+1)$ ! ways to complete $\mathcal{A} \cup \mathcal{A}^{\prime}$ to $F$.

Now we compute the number of ways to construct $\left(\mathcal{A}, \mathcal{A}^{\prime}\right)$ such that $\mathcal{A} \cap \mathcal{A}^{\prime}$ has a particular set of roots. This case will not exactly fit the structure of equations (39) and (40) and the discussion in that part of the proof, as we need to consider slightly different forests in the construction of $\mathcal{A} \backslash \mathcal{A}^{\prime}$ and $\mathcal{A}^{\prime} \backslash \mathcal{A}$.

Consider a forest $\mathcal{F}$ which may form the intersection $\mathcal{A} \cap \mathcal{A}^{\prime}$. We know that we must have at least 2 roots, $u$ being one of them, and that $v$ cannot belong to $\mathcal{F}_{u}$, if we are to ensure that $\mathcal{F}=\mathcal{A} \cap \mathcal{A}^{\prime}$ for the case $e \in \mathcal{A}, e \notin \mathcal{A}^{\prime}$. We will enumerate the forests satisfying this constraint by first contracting the arc $e$ from $u$ to $v$ (to signify its inclusion in $\mathcal{A}$ ). Consider the vertex set $V \backslash\{u\}$, and define the $\mathbf{s}, \mathbf{t}$ in-point and out-point vectors exactly as for case (i) above. Now let $R \subseteq V \backslash\{u\},|R| \geqslant 1$, and consider the set of forests $\mathcal{F}$ with root set $R$. By Lemma 2, the number of such forests is 1 if $R=V \backslash\{u\}$, and is

$$
\left(\prod_{w \in V \backslash\{u\} \cup R)} d_{w}\right)\left(\sum_{w \in R}\left(d_{w}+\left(d_{u}-2\right) \cdot \mathbb{1}_{w=v}\right)\right)(m-3)_{n-|R|-2}
$$

otherwise. The $\mathcal{F}$ forests do not represent possible configurations for $\mathcal{A} \cap \mathcal{A}^{\prime}$, given that we require $e \notin \mathcal{A}^{\prime}, e \in \mathcal{A}$. For any $\mathcal{F}$, let $r(v)$ be the element of $R$ which contains $v$. We will split the component $\mathcal{F}_{r(v)}$ into two subtrees $\mathcal{F}_{u}^{\prime}$ and $\mathcal{F}_{r(v)}^{\prime}$ (the latter containing $v$ ), by expanding $v$ into $u$ and $v$, assigning the appropriate $d_{u}-2$ in-points to $u$ and the other $d_{v}$ in-points to $v$, and finally deleting the arc $e$. This generates a slightly different forest $\mathcal{F}^{\prime}$ with root set $R=R \cup\{u\}$ such that $|R| \geqslant 1, u \notin R$ and such that $v \notin \mathcal{F}_{u}{ }^{\prime}$, as required. The split is uniquely done, and there is a one-to-one correspondence between forests $\mathcal{F}$ on $V \backslash\{u\}$ with root set $R$ and which have $e$ contracted in a particular component, and forests $\mathcal{F}^{\prime}$ on $V$ with root set $R \cup\{u\}$ and satisfying $v \notin V\left(\mathcal{F}_{u}{ }^{\prime}\right)$.

We now enumerate the number of ways we can extend $\mathcal{F}^{\prime}$ (which is the intersection $\left.\mathcal{A} \cap \mathcal{A}^{\prime}\right)$ to a pair of arborescences $\left(\mathcal{A}, \mathcal{A}^{\prime}\right)$ with the appropriate constraints. There are two stages; to choose the tree $\mathcal{A} \backslash \mathcal{A}^{\prime}$ on $\mathcal{F}$ which has root set $R$, and then to choose the tree $\mathcal{A}^{\prime} \backslash \mathcal{A}$ on $\mathcal{F}^{\prime}$ which has root set $R \cup\{u\}$. We define two vectors $\mathbf{x}, \mathbf{y}$ of length $|R|+1$ wrt the forest $\mathcal{F}^{\prime}$ on $V$. For every $w \in R \cup\{u\}, x_{w}$ denotes the number of points available for incoming arcs into component $\mathcal{F}_{w}^{\prime}$, and $y_{w}$ is the number of points available for outgoing points from $\mathcal{F}_{w}^{\prime}$. Observe that $y_{u}=d_{u}-2, y_{w}=d_{w}$ for all $w \in R$, and that

$$
x_{w}=\left(\sum_{z \in V\left(\mathcal{F}_{w}^{\prime}\right)} d_{z}\right)-\left|\mathcal{F}_{w}^{\prime}\right|+1-2 \cdot \mathbb{1}_{v \in V\left(\mathcal{F}_{w}^{\prime}\right)},
$$


for all $w \in R \cup\{u\}$. We note that the vectors $\mathbf{x}$ and $\mathbf{y}$ satisfy:

$$
\sum_{w \in R \cup\{u\}} x_{w}=\sum_{w \in R \cup\{u\}} y_{w}=m-n+|R|-1 .
$$

Now suppose we have chosen the arborescence $\mathcal{A} \backslash \mathcal{A}^{\prime}$ with root $r \in R$ on $\mathcal{F}$, and that this arborescence induces the child vector $\boldsymbol{\delta}$ counting the number of arcs from $\mathcal{A} \backslash \mathcal{A}^{\prime}$ directed into the components of $\mathcal{F}^{\prime}$. Now we consider the number of ways to choose $\mathcal{A}^{\prime} \backslash \mathcal{A}$, first considering the case $r^{\prime} \in(R \cup\{u\}) \backslash\{r\}$, then $r^{\prime}=r$. The relevant vectors for choosing $\mathcal{A}^{\prime} \backslash \mathcal{A}$ are $x_{w}-\delta_{w}$ for incoming arcs to $\mathcal{F}_{w}^{\prime}, w \in R \cup\{u\}$, and for outgoing arcs from $\mathcal{F}_{w}, y_{w}-1$ for all $w \in R \backslash\{r\}, y_{w}$ for $w \in\{r, u\}$ (we have $y_{u}$ here because the outgoing arc from $u$ in $\mathcal{A} \backslash \mathcal{A}^{\prime}$ is always $e$, and the outgoing point for $e$ has already been subtracted in defining $y_{u}$ ). We apply the same approach as in Theorem 3 . First we write the following expression (an analogue of (13) from Theorem 3) for the number of arborescences $\mathcal{A}^{\prime} \backslash \mathcal{A}$ rooted at $r^{\prime} \in R \cup\{u\}, r^{\prime} \neq r$ :

$$
\left(x_{r^{\prime}}-\delta_{r^{\prime}}\right) y_{r}\left(\prod_{w \in(R \cup\{u\}) \backslash\left\{r, r^{\prime}\right\}}\left(y_{w}-1 \cdot \mathbb{1}_{w \neq u}\right)\right)(m-n-1)_{|R|-1} .
$$

Similarly, we have the following expression (an analogue of (14)) for the number of arborescences $\mathcal{A}^{\prime} \backslash \mathcal{A}$ rooted at $r$ itself:

$$
\left(x_{r}-\delta_{r}\right)\left(\prod_{w \in(R \cup\{u\}) \backslash\{r\}}\left(y_{w}-1 \cdot \mathbb{1}_{w \neq u}\right)\right)(m-n-1)_{|R|-1} .
$$

Then weighting (45) by $\left(y_{r^{\prime}}-1 \cdot \mathbb{1}_{r^{\prime} \neq u}\right)\left(y_{r}-1\right)$ and (46) by $y_{r}\left(y_{r}-1\right)$ respectively, and summing over all $r^{\prime} \in R \cup\{u\}$, we obtain the value

$$
y_{r}\left(\prod_{w \in R \cup\{u\}}\left(y_{w}-1 \cdot \mathbb{1}_{w \neq u}\right)\right)(m-n)_{|R|}
$$

for the weighted sum of arbs $\mathcal{A}^{\prime} \backslash \mathcal{A}$, conditional on $\mathcal{A} \backslash \mathcal{A}^{\prime}$ having root $r$ and any child vector $\boldsymbol{\delta}$. Substituting in the values of the $y_{w}$, and using the fact that $r \neq u$, we have the value $\left(d_{u}-2\right) d_{r}\left[\prod_{w \in R}\left(d_{w}-1\right)\right](m-n)_{|R|}$.

Now let us count the number of configurations for $\mathcal{A} \backslash \mathcal{A}^{\prime}$ rooted at $r$. Given that (47) does not involve the value $\delta_{w}$ for any $w$, we will choose to sum over all child vectors, and to apply Lemma 2 directly, to obtain the total number of configurations for $\mathcal{A} \backslash \mathcal{A}^{\prime}$ with root $r$. This value is

$$
\left(\prod_{w \in R \backslash\{r\}} y_{w}^{\prime}\right) x_{r}^{\prime}\left(\sum_{w \in R} x_{v}^{\prime}-1\right)_{|R|-2},
$$

where the vectors $\mathbf{x}^{\prime}$ and $\mathbf{y}^{\prime}$ give the number of available points for incoming arcs, and for outgoing arcs respectively, for the vertices in $R$. We have $x_{w}^{\prime}=x_{w}$ and $y_{w}^{\prime}=y_{w}$ 
for $w \in R \backslash\{r(v)\}, x_{r(v)}^{\prime}=x_{r(v)}+x_{u}$ and $y_{r(v)}^{\prime}=y_{r(v)}$. Hence for every $w \in R, y_{w}^{\prime}=d_{w}$. Hence (48) can be re-written as

$$
x_{r}^{\prime}\left(\prod_{w \in R \backslash\{r\}} d_{w}\right)(m-n+|R|-2)_{|R|-2} .
$$

Now taking the product of (47) and (49), we have the following expression for the weighted sum over arborescences $\left(\mathcal{A} \backslash \mathcal{A}^{\prime}, \mathcal{A}^{\prime} \backslash \mathcal{A}\right.$ ), where $\mathcal{A} \backslash \mathcal{A}^{\prime}$ is rooted at $r$ (and subject to our constraints wrt $R$ and $e, f)$ :

$$
x_{r}^{\prime}\left(d_{u}-2\right)\left(\prod_{w \in R} d_{w}\left(d_{w}-1\right)\right)(m-n+|R|-2)_{2|R|-2} .
$$

We need to evaluate $\sum_{r \in R} x_{r}^{\prime}$, and by definition this is $\sum_{r \in R \cup\{u\}} x_{r}$, which by (44) is $(m-n+|R|-1)$. Therefore the sum of (50) over all $r \in R$ is

$$
\left(d_{u}-2\right)\left(\prod_{w \in R} d_{w}\left(d_{w}-1\right)\right)(m-n+|R|-1)_{2|R|-1} .
$$

Assuming for now that $R \neq V \backslash\{u\}$, we multiply by the number of forests on $V \backslash\{u\}$ with root set $R$, and by the number of ways $(m-n-|R|)$ ! of completing $\mathcal{A} \cup \mathcal{A}^{\prime}$ to a configuration of $\Phi_{n}^{\mathbf{d}}$, to obtain the expression

$$
\left(d_{u}-2\right)\left(\sum_{w \in R}\left(d_{w}+\left(d_{u}-2\right) \cdot \mathbb{1}_{w=v}\right)\right)\left(\prod_{w \in R}\left(d_{w}-1\right)\right)\left(\prod_{w \in V \backslash\{u\}} d_{w}\right)(m-3) ! .
$$

Athough we assumed $R \neq V \backslash\{u\}$ in order to derive this value, we get exactly the same expression for the case $R=V \backslash\{u\}$ by working with the value 1 for number of forests.

Finally, summing over all subsets $R \subseteq V \backslash\{u\}$, we obtain a value which is equal to $\left(d_{u}-2\right)\left(\prod_{w \in V \backslash\{u\}} d_{w}\right)(m-3)$ ! multiplied by

$$
\sum_{R \subseteq V \backslash\{u\}}\left(\sum_{w \in R}\left(d_{w}+\left(d_{u}-2\right) \cdot \mathbb{1}_{w=v}\right)\right)\left(\prod_{w \in R}\left(d_{w}-1\right)\right) .
$$

We apply the same approach as in Theorem 3, separating this expression into $(n-2)$ instances of $d_{w}\left(d_{w}-1\right) \sum_{R \subseteq V \backslash\{u, w\}} \prod_{z \in R}\left(d_{z}-1\right)$, one for each $w \in V \backslash\{v\}$. Each such instance evaluates to $\left(d_{w}-1\right) \prod_{z \in V \backslash\{v\}} d_{z}$. There is also an $(n-1)$-th expression $\left(d_{v}+d_{u}-2\right)\left(d_{v}-1\right) \prod_{z \in V \backslash\{u, v\}} d_{z}$. Adding all these, and multiplying again by $\left(d_{u}-2\right)\left(\prod_{w \in V \backslash\{u\}} d_{w}\right)(m-3)$ !, we obtain the value

$$
\left(m-n-1-\frac{d_{u}-2}{d_{v}}\right)\left(\frac{d_{u}-2}{d_{u}^{2}}\right)\left(\prod_{w \in V} d_{w}\right)^{2}(m-3) !
$$


which is the weighted sum over triples $\left(F, \mathcal{A}, \mathcal{A}^{\prime}\right)$ such that $e \in \mathcal{A}, e \notin \mathcal{A}^{\prime}$.

We obtain the same value for the other three cases where exactly one of $\mathcal{A}$ and $\mathcal{A}^{\prime}$ contains an element from $\{e, f\}$. Thus, the contribution to the weighted sum over triples $\left(F, \mathcal{A}, \mathcal{A}^{\prime}\right)$ from the case where exactly one of $\mathcal{A}$ and $\mathcal{A}^{\prime}$ contains an element from $\{e, f\}$ is, as $m-n \rightarrow \infty$,

$$
(m-n) \frac{4\left(d_{u}-2\right)}{d_{u}^{2}}\left(\prod_{w \in V} d_{w}\right)^{2}(m-3) ! .
$$

We now combine all three cases for (iii) to get an overall weighted sum over triples for $e, f$. When $d_{u}>2$, we take the sum of (42), (43), and (51), which is, as $m-n \rightarrow \infty$, asymptotically

$$
(m-n)\left(\prod_{w \in V} d_{w}\right)^{2}(m-3) ! .
$$

For $d_{u}=2$, our discussions for cases (ii) and (iii) imply that the weighted sum over triples is asymptotically equal to (42), and substituting $d_{u}=2$, this evaluates to (52) also.

We now consider the weights we apply to the triples $\left(F, \mathcal{A}, \mathcal{A}^{\prime}\right)$ from cases (i), (ii) and (iii). Define the degree vector $\mathbf{d}^{\prime}$ on $V$ by setting $d_{w}^{\prime}=d_{w}$ for $w \in V \backslash\{u\}$, and $d_{u}^{\prime}=d_{u}-2$. In almost all cases, a pair of arborescences $\left(\mathcal{A}, \mathcal{A}^{\prime}\right)$ with roots $r$ and $r^{\prime}, r \neq r^{\prime}$, will have been weighted by $\left(d_{r}^{\prime}-1\right)\left(d_{r^{\prime}}^{\prime}-1\right)$, and a pair which has the same root $r$ will have been weighted by factor $d_{r}^{\prime}\left(d_{r}^{\prime}-1\right)$. There is one exception: in case (iii), the pairs where one arborescence contains an arc from $\{e, f\}$ and is rooted at some $r^{\prime} \neq u$, and the other arborescence is rooted at $u$, have instead been multiplied by a factor of $d_{u}^{\prime}\left(d_{r^{\prime}}^{\prime}-1\right)$. This increases the value of (52) slightly over what we would obtain by applying $\left(d_{u}^{\prime}-1\right)\left(d_{r^{\prime}}^{\prime}-1\right)$. The extra contribution to $(52)$ for any $\operatorname{such}\left(F, \mathcal{A}, \mathcal{A}^{\prime}\right)$ is $\left(d_{r^{\prime}}-1\right) \leqslant(d-1)$. Summing over all $r^{\prime} \neq u$, and all such $\left(F, \mathcal{A}, \mathcal{A}^{\prime}\right)$, we can apply the BEST Theorem to show the total extra contribution is at most a $(d-1) / n$ fraction over the value we would have obtained for $(52)$ by weighting uniformly. Such a $(d-1) / n$ fraction is asymptotically negligible, hence from here on we will assume (52) was obtained by a uniform application of the weights.

Suppose $d_{u}>2$. By the BEST Theorem, for any particular Eulerian $F \in \Phi_{n}^{\mathbf{d}}$, the number of arborescences in $F$ is independent of the root. Assuming a uniform weighting of triples, the same argument as used for equation (22) of Theorem 3 shows we can correct for the weighting by dividing (52) by

$$
\frac{1}{n^{2}}(m-n-1)(m-n-2) \text {. }
$$

For the case of $d_{u}=2$, we have derived the expression (52) from case (i) triples alone (the contributions from (ii), (iii) being negligible), these being exactly those triples where neither arborescence is rooted at $u$. Summing the weights over pairs from $V \backslash\{u\}$, and dividing by $(n-1)^{2}$, we find that in the $d_{u}=2$ case, we can correct for the weighting in (42) by dividing by

$$
\frac{1}{(n-1)^{2}}(m-n)(m-n-1)
$$


In the limit, as $m-n \rightarrow \infty$, both (53) and (54) become $(m-n)^{2} / n^{2}$. Dividing (52) by $(m-n)^{2} / n^{2}$ and by the number of elements in $\widetilde{\Phi_{n}^{\mathbf{d}}}$ given by (23) gives

$$
\mathbb{P}\left[e, f \in F:\left(F, \mathcal{A}, \mathcal{A}^{\prime}\right) \in \widetilde{\Phi_{n}^{\mathbf{d}}}\right] \sim 1 / m^{2}
$$

This is the same probability for $e, f \in F$ when $F$ is chosen uniformly at random from $\Phi_{n}^{\mathrm{d}}$, so we can conclude

$$
\mathbb{E}\left[D^{(2)}\right] \sim \mathbb{E}[D]
$$

By following the same reasoning as was used with $L$ and $D$, we can show that the random variables $L^{(2)}$ and $D^{(2)}$ converge to (asymptotically) independent Poisson random variables. Hence, the probability that $F$ is simple, when $\left(F, \mathcal{A}, \mathcal{A}^{\prime}\right)$ is chosen uniformly at random from $\widetilde{\Phi_{n}^{\mathbf{d}}}$, satisfies

$$
\mathbb{P}\left[L^{(2)}=D^{(2)}=0\right] \sim \exp \left(-\frac{m_{2}-2 m+n}{m}-\frac{\left(m_{2}-m\right)^{2}}{2 m^{2}}\right) .
$$

Combining (30) and (55) gives the claimed estimate for $\mathbb{E}\left(\mathcal{A}_{n}\right)^{2}$.

Given the expectation and variance of the number of arborescences of a random $G \in$ $\mathcal{G}_{\mathrm{n}}^{\mathbf{d}}$, we can, from the BEST Theorem (Theorem 1), deduce the expectation and variance of the number of Euler tours of a uniformly random $G \in \mathcal{G}_{\mathrm{n}}^{\mathbf{d}}$.

Corollary 6. Let $d$ be some fixed constant, let $\mathbf{d}=\left(d_{1}, d_{2}, \ldots\right)$ be a sequence of positive integers satisfying $d_{i} \leqslant d$ for all $i$, let $n \in \mathbb{N}$, and let $m=\sum_{v=1}^{n} d_{v}$. Assume that $V_{1}$, the set of vertices $u$ such that $d_{u}=1$, satisfies the condition $\left|[n] \backslash V_{1}\right|=\Omega(n)$. Let $\mathcal{T}_{n}$ denote the number of Euler tours of a directed graph chosen randomly from $\mathcal{G}_{\mathrm{n}}^{\mathbf{d}}$. Then, as $m-n \rightarrow \infty$,

$$
\begin{aligned}
& \mathbb{E}\left[\mathcal{T}_{n}\right] \sim \frac{e}{m}\left[\prod_{v \in[n]}\left(d_{v}\right) !\right] \\
& \mathbb{E}\left[\mathcal{T}_{n}^{2}\right] \sim e^{-n / m} \frac{m}{m-n} \mathbb{E}\left[\mathcal{T}_{n}\right]^{2} .
\end{aligned}
$$

We now consider our estimates for the first and second moment in the context of Chebyshev's inequality, which states that for a random variable $X$ with expectation $\mu(X)$ and standard deviation $\sigma(X)$, that for any $k>0$, the probability that $X$ deviates by more than $k \sigma(X)$ from its mean is bounded as follows:

$$
\mathbb{P}[|X-\mu(X)| \geqslant k \sigma(X)] \leqslant \frac{1}{k^{2}}
$$

For us $X$ is $\mathcal{T}_{n}$, and by Corollary 6 ,

$$
\sigma\left(\mathcal{T}_{n}\right)^{2} \sim \mathbb{E}\left[\mathcal{T}_{n}\right]^{2}\left[\frac{e^{-n / m}}{(1-n / m)}-1\right], \quad \text { as } n \rightarrow \infty
$$


Using basic calculus, we can see that the function $e^{x} /(1+x)$ is strictly decreasing on the range $[-1,0]$, attaining a minimum value of 1 at $x=0$. Thus, whenever $m=c n$ for some fixed constant $c>1$, we can find a constant $b_{c}>0$ such that $\sigma\left(\mathcal{T}_{n}\right)^{2} \sim b_{c} \mathbb{E}\left[\mathcal{T}_{n}\right]^{2}$. For example, in the case $d_{v}=2$ for all $v$, we will have $\sigma\left(\mathcal{T}_{n}\right)^{2} / \mathbb{E}\left[\mathcal{T}_{n}\right]^{2} \sim\left(2 e^{-1 / 2}-1\right) \approx 0.213$.

Now suppose we want to use (56) to bound probability $\mathbb{P}\left[\mathcal{T}_{n} \leqslant n^{-\alpha} \mathbb{E}\left[\mathcal{T}_{n}\right]\right]$. Our goal must be to show that this probability tends to 0 as $n \rightarrow \infty$. The condition $\mathcal{T}_{n} \leqslant n^{-\alpha} \mathbb{E}\left[\mathcal{T}_{n}\right]$ is equivalent to $\mathbb{E}\left[\mathcal{T}_{n}\right]-\mathcal{T}_{n} \geqslant\left(1-n^{-\alpha}\right) \mathbb{E}\left[\mathcal{T}_{n}\right]$. Given the relationship between $\mathbb{E}\left[\mathcal{T}_{n}\right]$ and $\sigma\left(\mathcal{T}_{n}\right)$ above, this is also, as $n \rightarrow \infty$, asymptotically equivalent to $\mathbb{E}\left[\mathcal{T}_{n}\right]-\mathcal{T}_{n} \geqslant$ $\left(1-n^{-\alpha}\right) \sigma\left(\mathcal{T}_{n}\right) b_{c}^{-1 / 2}$. We now apply Chebyshev' inequality $(56)$ to bound $\mathbb{P}\left[\left|\mathbb{E}\left[\mathcal{T}_{n}\right]-\mathcal{T}_{n}\right| \geqslant\right.$ $\left.\left(1-n^{-\alpha}\right) \sigma\left(\mathcal{T}_{n}\right) b_{c}^{-1 / 2}\right]$. We have $k=\left(1-n^{-\alpha}\right) b_{c}^{-1 / 2}$, giving

$$
\mathbb{P}\left[\left|\mathbb{E}\left[\mathcal{T}_{n}\right]-\mathcal{T}_{n}\right| \geqslant\left(1-n^{-\alpha}\right) \sigma\left(\mathcal{T}_{n}\right) b_{c}^{-1 / 2}\right] \leqslant \frac{b_{c}}{\left(1-n^{-\alpha}\right)^{2}}
$$

Thus, the asymptotic upper bound on $\mathbb{P}\left[\mathcal{T}_{n} \leqslant n^{-\alpha} \mathbb{E}\left[\mathcal{T}_{n}\right]\right]$ that we obtain using (56) with Corollary 6 is at best $b_{c}$, which does not tend to 0 in general. Hence Chebyshev's inequality is not sufficient to allow us to show condition (b) of Subsection 1.2.

In the next section, we will show how to use results of this section and the estimates of Corollary 6 to obtain an asymptotic distribution for the number of Euler tours of a random $G \in \mathcal{G}_{\mathrm{n}}^{\mathrm{d}, \mathrm{d}}$, from which we can derive a stronger concentration result.

\section{Asymptotic distribution of Euler tours}

In this section we compute the asymptotic distribution of the number of Euler tours of a random $d$-in/d-out directed graph. The case $d=1$ is trivial, since the number of Euler tours of a random 1-in/1-out graph will be 0, with high probability. For this reason, and also because Theorem 5 and Corollary 6 require $m-n \rightarrow \infty$, we restrict our attention to the case $d \geqslant 2$.

To compute the asymptotic distribution we will use the following general theorem of Janson [6] (see also [7, Chapter 9]).

Theorem 7 (Janson [6]). Let $\lambda_{i}>0$ and $\delta_{i} \geqslant-1, i=1,2, \ldots$, be constants and suppose that for each $n$ there are random variables $X_{i n}, i=1,2, \ldots$, and $Y_{n}$ (defined on the same probability space) such that $X_{\text {in }}$ is non-negative integer valued and $\mathbb{E}\left[Y_{n}\right] \neq 0$ (at least for large $n$ ) and furthermore the following conditions are satisfied:

1. $X_{\text {in }} \stackrel{d}{\rightarrow} X_{i \infty}$ (in distribution) as $n \rightarrow \infty$, jointly for all $i$, where $X_{i \infty}$ is a Poisson random variable with mean $\lambda_{i}$;

2. For any finite sequence $x_{1}, \ldots x_{k}$ of non-negative integers

$$
\frac{\mathbb{E}\left[Y_{n} \mid X_{1 n}=x_{1}, \ldots X_{k n}=x_{k}\right]}{\mathbb{E}\left[Y_{n}\right]} \sim \prod_{i=1}^{k}\left(1+\delta_{i}\right)^{x_{i}} e^{-\lambda_{i} \delta_{i}} \quad \text { as } n \rightarrow \infty
$$


3. $\sum_{i} \lambda_{i} \delta_{i}^{2}<\infty$;

4. $\frac{\mathbb{E}\left[Y_{n}^{2}\right]}{\mathbb{E}\left[Y_{n}\right]^{2}} \sim \exp \left(\sum_{i} \lambda_{i} \delta_{i}^{2}\right)$;

Then

$$
\frac{Y_{n}}{\mathbb{E}\left[Y_{n}\right]} \stackrel{d}{\rightarrow} W=\prod_{i=1}^{\infty}\left(1+\delta_{i}\right)^{X_{i \infty}} e^{-\lambda_{i} \delta_{i}}
$$

Moreover, this and the convergence in (1) holds jointly. The infinite product defining $W$ converges a.s. and in $L_{2}$, with $\mathbb{E}[W]=1, \mathbb{E}\left[W^{2}\right]=\exp \left(\sum_{i} \lambda_{i} \delta_{i}^{2}\right)=\lim _{n \rightarrow \infty} \mathbb{E}\left[Y_{n}\right]^{2} / \mathbb{E}\left[Y_{n}\right]^{2}$. Hence, the normalised variables are uniformly square integrable. Furthermore, the event $W=0$ equals, up to a set of probability 0 , the event that $X_{i \infty}=0$ for some $i$ with $\delta_{i}=-1$. In particular, $W>0$ a.s. if and only if every $\delta_{i}>-1$.

In our application of Theorem 7 we will have $Y_{n}=\mathcal{T}_{n}$, the random variable counting Euler tours of $d$-in/d-out graphs, and $X_{\text {in }}$ equal to the number of directed $i$-cycles in a random $d$-in/d-out graph. To apply Theorem 7 we need the following two lemmas.

Lemma 8. For each positive integer $i$ let $X_{\text {in }}$ count the number of directed $i$-cycles in a directed graph obtained as the projection of a uniformly random $F \in \Phi_{n}^{d, d}$. The variables $X_{i n}$ are asymptotically independent Poisson random variables with means $\mathbb{E}\left[X_{i n}\right]=\lambda_{i}=$ $\frac{d^{i}}{i}$.

Proof. First, suppose $\mathbb{E}\left[X_{i n}\right] \sim \lambda_{i}$ for each $i$. Recall that the probability $\sigma(F)$ contains any particular subgraph $H$ with more arcs than vertices is negligible, when $F$ is chosen uniformly at random from $\Phi_{n}^{d, d}$ (Lemma 4). Hence, we can assume that cycles occur independently, i.e., for any sequence of non-negative integers $k_{1}, \ldots, k_{\ell}$, we have

$$
\mathbb{E}\left[\prod_{i=1}^{\ell}\left(X_{i n}\right)_{k_{i}}\right] \sim \prod_{i=1}^{\ell} \lambda_{i}^{k_{i}}
$$

Hence, the random variables $X_{1 n}, \ldots, X_{\ell n}$ converge to a set of independent Poisson random variables.

We will now show $\mathbb{E}\left[X_{i n}\right] \sim \lambda_{i}$. We say a set of $i$ edges $e_{1}, e_{2}, \ldots, e_{i}$ in a configuration is an $i$-cycle if there is a sequence of distinct vertices $v_{1}, v_{2}, \ldots, v_{i}$ such that $e_{j} \in S_{v_{j}} \times T_{v_{j+1}}$ for $j<i$ and $e_{i} \in S_{v_{i}} \times T_{v_{1}}$. The probability of any particular $i$-cycle being contained in a random $F \in \Phi_{n}^{d, d}$ is

$$
\frac{(d n-i) !}{(d n) !} \sim \frac{1}{(d n)^{i}} .
$$

So, to estimate $\mathbb{E}\left[X_{i n}\right]$ all we need to do is count the number of different $i$-cycles that can occur in some $F \in \Phi_{n}^{d, d}$ and then divide by $(d n)^{i}$. Let $I$ be some $i$-subset of $[n]$. There are $(i-1)$ ! different ways to arrange $I$ into an $i$-cycle $\left(v_{1}, v_{2}, \ldots, v_{i}\right)$ and then $d^{2 i}$ ways to choose edges $e_{j} \in S_{v_{j}} \times T_{v_{j+1}}$ for $1 \leqslant j<i$ and $e_{i} \in S_{v_{i}} \times T_{v_{1}}$. Hence,

$$
\mathbb{E}\left[X_{i n}\right] \sim \frac{(i-1) !}{(d n)^{i}}\left(\begin{array}{c}
n \\
i
\end{array}\right) d^{2 i},
$$

and so $\mathbb{E}\left[X_{i n}\right] \sim \lambda_{i}$ 
Lemma 9. Let $X_{\text {in }}$ be as in Lemma 8 and let $\mu_{i}=\frac{d^{i}-1}{i}$. Then, for any fixed set of non-negative integers $k_{1}, k_{2}, \ldots, k_{\ell}$ we have

$$
\frac{\mathbb{E}\left[\mathcal{A}_{n}^{\star} \prod_{i=1}^{\ell}\left(X_{i n}\right)_{k_{i}}\right]}{\mathbb{E}\left[\mathcal{A}_{n}^{\star}\right]} \sim \prod_{i=1}^{\ell} \mu_{i}^{k_{i}}
$$

Proof. We only verify

$$
\frac{\mathbb{E}\left[\mathcal{A}_{n}^{\star} X_{i n}\right]}{\mathbb{E}\left[\mathcal{A}_{n}^{\star}\right]} \sim \mu_{i} ;
$$

convergence of the factorial moments holds for the same reasons as were given in Lemma 8 .

Let $\overline{\Phi_{n}^{d, d}}$ be the set defined in (7) (for the case $d_{v}=d$ for all $v$ ) and let $I$ be an $i$-subset of $[n]$. As in the previous lemma, there are $(i-1) ! d^{2 i}$ ways to choose a configuration for an $i$-cycle on $I$. To estimate $\mathbb{E}\left[\mathcal{A}_{n}^{\star} X_{i n}\right]$ we need to calculate the probability that a particular $i$-cycle $C$ is contained in $F$ when $(F, \mathcal{A})$ is chosen uniformly at random from $\overline{\Phi_{n}^{d, d}}$. Suppose $C \cap \mathcal{A}$ has $c$ components, $P_{1}, P_{2}, \ldots, P_{c}$, each of which is a directed path, and let $v_{j}$ be the final vertex in the path $P_{j}$ for $1 \leqslant j \leqslant c$. Choosing the remainder of $\mathcal{A}$ is then equivalent to choosing an arborescence on $(V \backslash I) \cup\left\{v_{j}: 1 \leqslant j \leqslant c\right\}$, where we have collapsed each path to a single vertex. Each $v \in V \backslash I$ has $d$ points available for arcs directed towards or away from $v$. For each $j=1 \ldots c$, there are $\left|P_{j}\right|(d-1)$ points available for arcs directed towards $v_{j}$, and $d-1$ points available for arcs directed away from $v_{j}$. Once we have chosen $\mathcal{A}$, there are $(d n-n-c+1)$ ! ways to complete $F$. Hence, using Lemma 2, we can deduce that the number of ways to choose the remainder of $(F, \mathcal{A})$ is

$$
n(d-1)^{c} d^{n-i}(d n-i-1) !
$$

Summing over all the possible choices for $P=\left\{v_{1}, v_{2}, \ldots, v_{c}\right\}$, multiplying by $\left(\begin{array}{c}n \\ i\end{array}\right)$ (the number of ways to choose $I$ ) and $(i-1) ! d^{2 i}$ (the number of ways to choose configurations for a set of cycles on $I$ ), and finally dividing by $\left|\Phi_{n}^{d, d}\right|=(d n)$ !, gives

$$
\mathbb{E}\left[\mathcal{A}_{n}^{\star} X_{i n}\right]=\frac{\left(d^{i}-1\right)}{i} \frac{n d^{n+i}(n)_{i}}{(d n)_{i+1}}
$$

Letting $n \rightarrow \infty$, with $i$ fixed, in (57), we see that $\mathbb{E}\left[\mathcal{A}_{n}^{\star} X_{i n}\right] \sim \mu_{i} d^{n-1}$.

Corollary 10. Let $d \geqslant 2$ be some fixed integer, and let $\mathcal{T}_{n}$ denote the number of Euler tours in a directed graph $G$ chosen uniformly at random from $\mathcal{G}_{\mathrm{n}}^{\mathrm{d}, \mathrm{d}}$. For any fixed set of non-negative integers $k_{2}, \ldots, k_{\ell}$ we have

$$
\frac{\mathbb{E}\left[\mathcal{T}_{n} \prod_{i=2}^{\ell}\left(X_{i n}\right)_{k_{i}}\right]}{\mathbb{E}\left[\mathcal{T}_{n}\right]} \sim \prod_{i=2}^{\ell} \mu_{i}^{k_{i}}
$$

We are now able to apply Janson's theorem to obtain an asymptotic distribution for the number of Euler tours of a uniformly random $G \in \mathcal{G}_{\mathrm{n}}^{\mathrm{d}, \mathrm{d}}$. 
Theorem 11. Let $d \geqslant 2$ be some fixed integer, and let $\mathcal{T}_{n}$ denote the number of Euler tours in a directed graph $G$ chosen uniformly at random from $\mathcal{G}_{\mathrm{n}}^{\mathrm{d}, \mathrm{d}}$. Then,

$$
\frac{\mathcal{T}_{n}}{\mathbb{E}\left[\mathcal{T}_{n}\right]} \stackrel{d}{\rightarrow} \prod_{i=2}^{\infty}\left(1-\frac{1}{d^{i}}\right)^{Z_{i}} e^{1 / i}
$$

where the $Z_{i}$ are independent Poisson random variables with means $d^{i} / i$.

Proof. It suffices to show that conditions (1) to (4) of Theorem 7 are satisfied by $\mathcal{T}_{n}$ and $\left\{X_{i n}: i \geqslant 2\right\}$, where $X_{i n}$ is the random variable counting $i$-cycles. Lemma 8 and Corollary 10 provide conditions (1) and (2) with

$$
\lambda_{i}=\frac{d^{i}}{i} \quad \text { and } \quad \delta_{i}=-\frac{1}{d^{i}} .
$$

With these values, evaluating the sum in condition (3) gives

$$
\sum_{i=2}^{\infty} \frac{1}{i d^{i}}=-\frac{1}{d}+\log \left(\frac{d}{d-1}\right) .
$$

Finally, Corollary 6 provides condition (4).

\section{Generating and counting Euler tours}

We now turn to the analysis of Algorithm SAmple in Section 1. Note that although the algorithm is defined in terms of transition systems, it can also be considered as equivalent to a random directed walk on the Eulerian digraph or graph; terminating when we have used all outgoing edges of the starting vertex (whether we have created an Euler tour or not). This procedure was first considered in [8], where the authors considered it for undirected graphs and showed that the expected number of runs needed to obtain an Euler tour is polynomial for the case of $G=K_{n}$ for odd $n$. We consider the algorithm for $G \in \mathcal{G}_{\mathrm{n}}^{\mathrm{d}, \mathrm{d}}$ and are interested in the quantity

$$
\frac{|E T(G)|}{(d !)^{n}} .
$$

The following theorem uses the results of the previous section (by a similar argument to that used in [5, Lemma 1]) to show that this value is $\Omega\left(n^{-2}\right)$ with high probability when $G$ is chosen uniformly at random from $\mathcal{G}_{\mathrm{n}}^{\text {d,d }}$. When this is the case, we can generate uniformly random Euler tours of $G$ in expected polynomial time. Moreover, by setting the value of $\kappa$ in Approximate appropriately, we can approximate $|E T(G)|$.

Theorem 12. Let $d$ be some fixed integer, $d \geqslant 2$, and let $G$ be chosen uniformly at random from $\mathcal{G}_{\mathrm{n}}^{\mathrm{d}, \mathrm{d}}$. Then,

as $n \rightarrow \infty$.

$$
\mathbb{P}\left[\frac{|E T(G)|}{(d !)^{n}} \in \Omega\left(n^{-2}\right)\right] \rightarrow 1
$$


Proof. We first note that by the estimate for $\mathbb{E}\left[\mathcal{T}_{n}\right]$ given in Corollary 6 , it suffices to show that

$$
\mathbb{P}\left[\mathcal{T}_{n} \geqslant n^{-1} \mathbb{E}\left[\mathcal{T}_{n}\right]\right] \rightarrow 1
$$

For $\mathbf{x}=\left(x_{2}, \ldots, x_{k}\right)$ we define $\mathcal{G}_{\mathbf{x}}$ to be the set of all $d$-in/d-out graphs containing exactly $x_{i}$ directed cycles of length $i$ for each $i=2 \ldots k$, and

$$
W(\mathbf{x})=\prod_{i=2}^{k}\left(1-\frac{1}{d^{i}}\right)^{x_{i}} e^{1 / i}
$$

For each fixed $\gamma>0$ we define

$$
S(\gamma)=\left\{\mathbf{x}: x_{i} \leqslant \lambda_{i}+\gamma \lambda_{i}^{2 / 3} \text { for } 2 \leqslant i \leqslant k\right\} .
$$

From Lemma 8 (and Lemma 3 of [13]), we can deduce that the probability of a random $d$-in/d-out graph $G$ not being contained in $\mathcal{G}_{\mathbf{x}}$ for some $\mathbf{x} \in S(\gamma)$ is $O\left(e^{-a \gamma}\right)$, where $a$ is an absolute constant independent of $\gamma$. Hence, to verify the theorem all we need do is show that

$$
W(\mathbf{x}) \geqslant e^{-(b+c) \gamma} \quad \forall \mathbf{x} \in S(\gamma),
$$

where $b$ and $c$ are absolute constants independent of $\gamma$. For any particular $b, c$ and $\gamma$, we can choose $n$ sufficiently large so that $e^{-(b+c \gamma)} \geqslant n^{-1}$. Then, if (60) holds, we have

$$
\mathbb{P}\left[\mathcal{T}_{n} \geqslant n^{-1} \mathbb{E}\left[\mathcal{T}_{n}\right]\right] \geqslant 1-O\left(e^{-a \gamma}\right) .
$$

The above holds for any constant $\gamma$, and so can be taken as equal to 1 in the limiting case.

So, it remains to prove (60). For $\mathbf{x} \in S(\gamma)$ we have $W(\mathbf{x}) \geqslant A B^{\gamma}$, where

$$
\begin{aligned}
& A=\prod_{i \geqslant 2}\left(1-\frac{1}{d^{i}}\right)^{\lambda_{i}} e^{1 / i} \\
& B=\prod_{i \geqslant 2}\left(1-\frac{1}{d^{i}}\right)^{\lambda_{i}^{2 / 3}} .
\end{aligned}
$$

We can bound the right hand side of (61) as

$$
A \geqslant \prod_{i=2}^{\infty} \exp \left(\frac{1}{i}-\frac{d^{i}}{i\left(d^{i}-1\right)}\right)=\exp \left(\sum_{i=2}^{\infty}-\frac{1}{i\left(d^{i}-1\right)}\right) .
$$

The sum inside the exponential is clearly convergent, so we can conclude that $A \geqslant e^{-b}$ for some absolute constant $b$. Similarly, we can bound $B$ by

$$
B \geqslant \exp \left(-\sum_{i=2}^{\infty} \frac{1}{i^{2 / 3}\left(d^{i / 3}-1\right)}\right)
$$

and, again, the sum in the exponential is convergent, so $B^{\gamma} \geqslant e^{-c \gamma}$ for some absolute constant $c$. 


\section{Acknowledgements}

This work has benefited from conversations with Kyriakos Kalorkoti. The original idea for applying Robinson and Wormald's technique to the number of Euler tours came from Alan Frieze.

\section{References}

[1] Richard Arratia, Béla Bollobás, Don Coppersmith, and Gregory B. Sorkin. Euler circuits and DNA sequencing by hybridization. Discrete Applied Mathematics, 104(13):63-96, 2000.

[2] Edward A. Bender and E. Rodney Canfield. The Asymptotic Number of Labeled Graphs with Given Degree Sequences. Journal of Combinatorial Theory, Series A, 24(3):296-307, 1978.

[3] Béla Bollobás. A probabilistic proof of an asymptotic formula for the number of labelled regular graphs. European Journal of Combinatorics, 1:311-316, 1980.

[4] Charles J. Colbourn, Wendy J. Myrvold, and Eugene Neufeld. Two Algorithms for Unranking Arborescences. Journal of Algorithms, 20(2):268-281, 1996.

[5] Alan M. Frieze, Mark Jerrum, Michael Molloy, Robert W. Robinson, and Nicholas C. Wormald. Generating and Counting Hamilton Cycles in Random Regular Graphs. Journal of Algorithms, 21(1):176-198, 1996.

[6] Svante Janson. Random Regular Graphs: Asymptotic Distributions and Contiguity. Combinatorics, Probability \& Computing, 4:369-405, 1995.

[7] Svante Janson, Tomasz Łuczak, and Andrzej Riciński. Random Graphs. Wiley, 2000.

[8] Brendan D. McKay and Robert W. Robinson. Asymptotic Enumeration of Eulerian Circuits in the Complete Graph. Combinatorics, Probability \& Computing, 7(4):437449, 1998.

[9] Michael S. O. Molloy, Hanna D. Robalewska, Robert W. Robinson, and Nicholas C. Wormald. 1-Factorizations of random regular graphs. Random Structures and Algorithms, 10(3):305-321, 1997.

[10] James Gary Propp and David Bruce Wilson. How to Get a Perfectly Random Sample From a Generic Markov Chain and Generate a Random Spanning Tree of a Directed Graph. Journal of Algorithms, 27:170-217, 1998.

[11] Hanna D. Robalewska. 2-factors in random regular graphs. Journal of Graph Theory, 23(3):215-224, 1996.

[12] Robert W. Robinson and Nicholas C. Wormald. Almost All Cubic Graphs Are Hamiltonian. Random Structures \& Algorithms, 3(2):117-126, 1992.

[13] Robert W. Robinson and Nicholas C. Wormald. Almost All Regular Graphs Are Hamiltonian. Random Structures \& Algorithms, 5(2):363-374, 1994. 
[14] C. A. B. Smith and W. T. Tutte. On unicursal paths in networks of degree 4. American Mathematical Monthly, 48:233-237, 1941.

[15] Richard P. Stanley. Enumerative Combinatorics, volume 2. Cambridge University Press, 2001.

[16] W.T. Tutte. Graph Theory, Encyclopedia of Mathematics and its Applications, volume 21. Addison-Wesley Publishing Company, 1984.

[17] Tanja van Aardenne-Ehrenfest and Nicholas G. de Bruijn. Circuits and trees in oriented linear graphs. Simon Stevin, 28:203-217, 1951.

[18] Virginia Vassilevska Williams. Multiplying matrices faster than coppersmithwinograd. Proceedings of the Symposium on Theory of Computing (STOC 2012), 887-898, 2012. 